\author{
AUTHOR'S POST PRINT (Romeo Colour: Green) \\ Int. J. Num. Meth. Heat Fluid Flow (ISSN: 0961-5539), 10 (7): 721-749 (2000). \\ DOI: $10.1108 / 09615530010350444$ \\ Publisher version available at \\ http://www.emeraldinsight.com/journals.htm?articleid=877202\&show=abstract
}

\title{
Influence of buoyancy forces on Marangoni flow instabilities in liquid bridges
}

\author{
M. Lappa*, R. Savino and R. Monti \\ Università degli Studi di Napoli "Federico II" \\ Dipartimento di Scienza e Ingegneria dello Spazio "Luigi G. Napolitano" \\ P.le V.Tecchio 80, 80125 Napoli (Italy) \\ *current e-mail address: marcello.lappa@strath.ac.uk
}

\begin{abstract}
The influence of buoyancy forces on oscillatory Marangoni flow in liquid bridges of different aspect ratio is investigated by three-dimensional, time-dependent numerical solutions and by laboratory experiments using a micro-scale apparatus and a thermographic visualisation system. Liquid bridges heated from above and from below are investigated. The numerical and experimental results show that for each aspect ratio and for both the heating conditions, the onset of the Marangoni oscillatory flow is characterized by the appearance of a standing wave regime; after a certain time, a second transition to a travelling wave regime occurs. The three-dimensional flow organization at the onset of instability is different according to whether the bridge is heated from above or from below. When the liquid bridge is heated from below, the critical Marangoni number is larger, the critical wave number $(\mathrm{m})$ is smaller and the standing wave regime is more stable, compared with the case of bridge heated from above. For the critical azimuthal wave number, two correlation laws are found as function of the geometrical aspect ratio A.
\end{abstract}




\section{Introduction}

The stability of free convection in non isothermal liquid bridges with quasi-cylindrical free surfaces has been the subject of intense research. These studies are motivated by the fact that flow instabilities in such configurations may be responsible for the appearance of undesirable striations in crystals grown by floating zone technique.

There are two types of convection in the floating zone: buoyancy and surface tension-driven convection. On the earth buoyancy convection arises, in the presence of the gravity field, due to density gradients induced by temperature differences applied to the liquid zone. In the microgravity environment provided by drop shaft or drop towers, sounding rockets or space stations, or in microscale experimentation (if the liquid volume is small enough) Marangoni flows, induced by the temperature dependence of the surface tension along the free surface of the zone, prevail over buoyancy convection.

Typically Marangoni convection is a surface phenomenon and is confined to regions near the free surface, whereas buoyancy drives fluid motion in the bulk of the specimen. Buoyancy forces are in fact "volume" driving actions, whereas Marangoni stresses are "surface" driving actions. It has been demonstrated that Marangoni convection may be subject to instability (Schwabe, Scharmann, Preisser and Oeder [1]). This instability may produce undesirable striations in the properties of the crystals produced in microgravity conditions.

In the last two decades a number of experimental works have been performed on the subject by different investigators [2-8]. These studies were carried out on ground through direct observation of the flow pattern inside the liquid bridge (transparent, high Prandtl number liquids were employed).

Due to the existence of temperature disturbances on the free surface of the liquid zone, the problem was also investigated in literature using thermographic techniques. This method was pionered by Monti et al.[9-10] who were the first to apply this technique to the free surface of a liquid. More recently, Muehlner et al.[11] have investigated the time-dependent features of the instability on ground using a thermographic system to visualize directly the temperature field. They used an infrared thermocamera with wavelengths centered at $4.61 \mathrm{~mm}$. Since in the wavelength band centered at $4.61 \mathrm{~mm}$ the substance they used $(\mathrm{Pr}=35)$ is partially transparent, these authors observed an average temperature distribution over a depth of about $70 \%$ of the radius of the liquid bridge so that only qualitative results were obtained. 
A number of studies have also appeared in which the problem was posed in the framework of linear stability theory to define sufficient conditions for instability in the non-dimensional parameter space [1215]. On the other hand, the development of supercomputers and of efficient numerical methods led the investigators to study the problem through numerical solution of the three-dimensional, non-linear and time-dependent Navier Stokes equations [16-19] which provides information on the features of the supercritical thermo-fluid-dynamic fields also far from the bifurcation point.

It was pointed out [17-18] that for high Prandtl number liquids and unitary aspect ratio, the instability of Marangoni flow is characterized by the appearance of a standing wave instability that, after a certain time, exhibits a second transition to a travelling wave model. This behaviour was confirmed from an experimental point of view by Monti, Savino, Lappa and Fortezza [19] and Shevtsova et al. [20].

In the standing wave regime, maximum and minimum surface temperature disturbances pulsate at fixed azimuthal positions. In the travelling wave regime the disturbances rotate in the azimuthal direction around the axis of the liquid zone.

In the present paper the influence of buoyancy effects on the critical Marangoni number, on the critical frequency, and on the critical azimuthal wave number as well as on the supercritical flow field (pulsating or rotating) is investigated by numerical simulations performed with a 3D numerical code based on control volume methods and non-uniform grids. Moreover, the numerical simulations are associated to experimental analyses performed using microscale apparatus and a thermographic diagnostic system able to provide direct visualization of the surface temperature spots induced by the Marangoni flow instability on the microzone surface; a thermocamera with wavelengths $8-12 \mathrm{~mm}$ has in fact been employed that does not make the silicone oil transparent allowing thus an investigation of the surface behaviour of the temperature field.

The problem related to the influence of buoyancy forces on the features of oscillatory Marangoni convection is particularly important during microscale experimentation on the ground, where gravity and thermocapillary effects are both present.

An excellent analysis of this problem has been given by the experimental work of Velten, Schwabe and Scharmann [4]. They studied the influence of gravity on the transition point, frequency and spatial structure of the flow field obtaining a large experimental data base. It was observed that the gravity effects can influence both the supercritical spatial organization and the occurrence of the oscillatory regime (pulsating or rotating). This problem has been considered from a numerical point of view 
partially in the linear stability analyses of Neitzel et al.[13] for $\operatorname{Pr}=1$, and of Kuhlman and Rath [14] for $\mathrm{Pr}=4$, whose results gave different stability boundaries. More recently, Kuhlmann et al. [21] and Wanschura et al. [22] have considered the combined buoyant-thermocapillary flow for a fixed aspect ratio using linear stability technique.

In the present paper the influence of the gravity field (heating from above or from below) on the critical azimuthal wave number is studied both numerically (numerical solution of the non linear and timedependent Navier Stokes equations) and experimentally (microscale apparatus) for different aspect ratios. A "physical explanation" of the influence of gravity effects on the features of Marangoni flow instability (still absent in literature) is given and a new interpretation of the results obtained by Velten, Schwabe and Scharmann [4] is provided.

\section{Physical and mathematical model}

Fig. 1 shows the geometry of the problem and the boundary conditions. A cylindrical liquid bridge is suspended between two coaxial disks with constant temperatures $\left(\bar{T}=\bar{T}_{O} \pm \Delta T / 2\right)$, where $\bar{T}_{0}$ is the ambient temperature and $\mathrm{T}$ the overall temperature difference. The geometrical aspect ratio of the bridge is defined as $\mathrm{A}=\mathrm{L} / \mathrm{D}$.

The liquid is assumed homogeneous and Newtonian, with constant density and transport coefficients. The bridge is bounded by a cylindrical and undeformable liquid-gas interface with a surface tension $\sigma$ exhibiting a linear decreasing dependence on the temperature: $\quad \sigma=\sigma_{0}-\sigma_{\mathrm{T}}\left(\overline{\mathrm{T}}-\overline{\mathrm{T}}_{0}\right)$ where $\sigma_{\mathrm{o}}$ is the surface tension for $\overline{\mathrm{T}}=\overline{\mathrm{T}}_{0} ; \sigma_{\mathrm{T}}$ is the negative rate of change of the surface tension with temperature $\left(\sigma_{\mathrm{T}}=-\mathrm{d} \sigma / \mathrm{d} \overline{\mathrm{T}}>0\right)$.

The Boussinesque approximation is used to model the buoyancy forces.

With the above assumptions the flow is governed by the continuity, Navier-Stokes and energy equations, that in non-dimensional conservative form read :

$\underline{\nabla} \cdot \underline{\mathrm{V}}=0$

$\frac{\partial \underline{\mathrm{V}}}{\partial \mathrm{t}}=-\underline{\nabla \mathrm{p}}-\underline{\nabla} \cdot[\underline{\mathrm{V}} \underline{\mathrm{V}}]+\operatorname{Pr} \nabla^{2} \underline{\mathrm{V}}-\operatorname{Pr} \mathrm{Ra} \mathrm{Ti}_{\underline{\mathrm{g}}}$

$\frac{\partial \mathrm{T}}{\partial \mathrm{t}}=-\underline{\nabla} \cdot[\underline{\mathrm{VT}}]+\nabla^{2} \mathrm{~T}$ 
where $\mathrm{V}, \mathrm{p}$ and $\mathrm{T}$ are the non-dimensional velocity, pressure and temperature, Pr is the Prandtl number, defined by $\operatorname{Pr}=v / \alpha$ ( $v$ is the kinematic viscosity and $\alpha$ the thermal diffusivity). The Rayleigh number is defined by $\mathrm{Ra}=\frac{\mathrm{g} \beta_{\mathrm{T}} \Delta \mathrm{TL}^{3}}{v \alpha}$ where $\beta_{\mathrm{T}}$ is the thermal expansion coefficient. The non-dimensional form results from scaling the lengths by the axial distance between the circular disks (L) and the velocity by the energy diffusion velocity $V_{\alpha}=\alpha / L$; the scales for time and pressure are, respectively, $L 2 / \alpha$ and $\rho \alpha^{2} / L^{2}$. The temperature, measured with respect to the initial temperature $\overline{\mathrm{T}}_{0}$, is scaled by $(\Delta \mathrm{T}): \mathrm{T}=$ $\left(\overline{\mathrm{T}}-\overline{\mathrm{T}}_{0}\right) /(\Delta \mathrm{T})$. The initial conditions are:

$\mathrm{t}=0: \quad \underline{\mathrm{V}}(\mathrm{z}, \mathrm{r}, \varphi)=0, \quad \mathrm{~T}(\mathrm{z}, \mathrm{r}, \varphi)=0$

For $\mathrm{t}>0$, the boundary conditions are the non-slip conditions and the condition of prescribed temperatures on the circular disks, the kinematic condition of stream surface (zero normal velocity), the Marangoni conditions (shear stress balance) and the adiabatic condition on the cylindrical interface. Further details on the model are reported in Ref.[18-19].

\section{Numerical solution}

\subsection{Solution method}

The equations (1a-c) and the initial and boundary conditions were solved numerically in cylindrical coordinates in primitive variables by a control volume method. The domain was discretized with a non uniform but structured axisymmetric mesh and the flow field variables defined over a staggered grid.

The finite volume approach relies directly on the application of the integral form of balance laws. Thus the conservation laws have been written for an arbitrary spatial domain $\Omega$ bounded by a surface $\partial \Omega$. Forward differences in time were used to discretize the time dependent derivative, obtaining:

$$
\begin{aligned}
& \underline{V}^{n+1}=\underline{V}^{n}+\Delta t \frac{1}{\Omega}\left[-\int_{\Omega \Omega}[\underline{V} \underline{V}] \cdot \underline{n} d S+\operatorname{Pr} \int_{\Omega \Omega}[\underline{\nabla} \underline{V}] \cdot \underline{n} d S-\operatorname{Pr} \operatorname{Ra} \dot{i}_{\underline{\mathrm{g}}} \int_{\Omega} T d \Omega\right]^{n}-\Delta t \frac{1}{\Omega} \int_{\Omega} \underline{\nabla} p^{n} d \Omega \\
& T^{n+1}=T^{n}+\Delta t \frac{1}{\Omega}\left[-\int_{\Omega \Omega}[V T] \cdot \underline{n} d S+\int_{\Omega}[\nabla T] \cdot \underline{n} d S\right]^{n}
\end{aligned}
$$

The problem is solved with the well known Marker and Cell method on a parallel supercomputer (see Lappa and Savino [23]). 


\subsection{Validation of the numerical procedure}

The numerical model has been validated by quantitative comparisons with $2 \mathrm{D}$ numerical results and with existing three-dimensional linear stability results for Prandtl numbers as close to the one used in the present work as possible.

For two-dimensional computations, tables $\mathrm{Ia}$ and $\mathrm{Ib}$ show that values obtained with the present code compose extremely well with those by Kuhlmann and Rath [14] and Wanschura et al.[15].

A grid refinement study has been also performed to show the numerical convergence of the present algorithm. The computations have been performed for uniform grids $\mathrm{Nz} \times \mathrm{Nr}$ (the first number denotes the number of collocation points in the axial direction, and the second define the grid size in the radial direction) and for non-uniform grids. For the latter a finer grid has been introduced near the free surface to locally enhance the resolution. The number of points clustered near the free surface are specified using the notation $\mathrm{Nz} \times\left(\mathrm{Nr}_{\mathrm{b}}+\mathrm{Nr}_{\mathrm{s}}\right.$ ) (where $\mathrm{Nr}_{\mathrm{s}}$ is the number of points stretched near the free surface and $\mathrm{Nr}_{\mathrm{b}}$ the points uniformly distributed in the bulk over a radius $\mathrm{R}_{b}=0.7 \mathrm{R}$ ). $T$ he $\mathrm{Nr}_{\mathrm{s}}$ points near the free surface are clustered using the stretching function (s) due to Roberts and Eiseman (for further details see Fletcher [24]; for the present computations the stretching control parameters $P=1$ and $Q=2$ have been used). This corresponds to a grid stretching factor $\delta$ not constant with a maximum value $\left(\delta_{\max }\right)$ for the layer of points adjacent the free surface.

To check that the code is able to "capture" the physical instabilities of Marangoni flow, critical Marangoni numbers have been computed and compared with the results of available stability analyses. In particular the case $\mathrm{A}=0.5, \mathrm{Pr}=4$ (reported in the linear stability analysis of Wanschura et al. [15]) has been considered.

Regarding the definition of the critical conditions for the onset of instability, it is important to clarify the criterion adopted to evaluate the so-called critical Marangoni number.

The linear stability analyses assume that the critical Marangoni number $\mathrm{Ma}_{\mathrm{c}}$ is a threshold value of the Marangoni number at which the growth rate of the disturbances is zero. Typically, in these analyses the critical Marangoni number is computed using an extrapolation of the growth rate to zero. For the present calculations, the critical Marangoni numbers have been defined as those for which the disturbance amplitude becomes vanishing (i.e. the non-dimensional azimuth velocity is of the order of $10^{-2}$ ), but is 
still characterized by a well-defined frequency. These values have been determined using direct 3D simulation rather than extrapolation.

For $\operatorname{Pr}=4$ and $A=0.5$ Wanschura et al.[15] predict $m=2$ and $\mathrm{Ma}_{\mathrm{c}} \cong 4200$. The present results give $\mathrm{Ma}_{\mathrm{c}} \cong 4400(5 \%$ greater that the linear stability value) and a pulsating regime that develops in a travelling regime after a short transient time. The grid refinement study has been conducted also on the influence of the number of points used in azimuthal direction (Table Id).

Preliminary numerical tests performed with the code on different computers have shown that the timedelay for transition from standing to travelling wave depends upon the numerical noise related to the machine used for the simulations. For this reason one should expect differences between the numerical results related to the time-delays presented in the following paragraphs and the times occurring experimentally.

\section{Experimental procedure}

The experimental facility used to perform the experiments includes a mechanical support system, a temperature control system and a visualisation system.

The support system consists of two cylindrical copper rods, equipped with Peltier elements, which sustain the bridge. The two rods have the same diameter $(\mathrm{D}=4[\mathrm{~mm}])$.

The upper disk can be translated along the vertical axis in order to realize a bridge having the desired aspect ratio. The translation is obtained using a computer-controlled motorized micrometer system.

The temperature control system, which is connected with a tension generator, includes two Peltier elements that establish appropriate temperature on the disks in order to impose the desired temperature differences. The active cooling and heating system is designed in order to reduce the heat transfer from the oil to the ambient. The system, based on the Peltier elements, allows the temperature ramps of the two disks to be controlled imposing symmetric temperature profiles with respect to the initial temperature.

Surface temperature distribution is measured by an infrared thermocamera (it is possible to visualize an extension of the free surface corresponding to an angular range of 160 degrees) capable of aquiring 12 fps. The infrared thermocamera makes it possible to obtain direct visualization of the temperature spots on the surface of the bridge that correspond to the minimum and maximum temperature disturbances.

During an experiment, typically the temperature difference between supports is varied heating one disk and cooling the other one, with respect to the ambient temperature, with symmetrical temperature ramps 
and with a very slow ramping rate. When the fully established oscillatory regime is reached, the temperature distribution on the liquid bridge surface is visualized using the infrared thermocamera.

The thermocamera provides direct visualization of the temperature distribution on the surface of the bridge. The temperature disturbances are computed in a post-analysis phase by subtracting from the surface temperature distribution the time-averaged surface temperature field $T_{0}(z, \varphi)$ obtained integrating the experimentally measured surface temperature distribution over the period $\tau$ of the oscillations.

$$
\mathrm{T}_{\mathrm{O}}(\mathrm{z}, \varphi)=\frac{1}{\tau} \int_{0}^{\tau} \mathrm{T}(\mathrm{z}, \varphi, \mathrm{t}) \mathrm{dt}=\frac{1}{\mathrm{~N}} \sum_{\mathrm{i}=1}^{\mathrm{N}} \mathrm{T}_{\mathrm{i}}(\mathrm{z}, \varphi)
$$

where $\mathrm{N}$ is the number of images taken during the period $\tau$.

\section{Results and discussion}

Parametric numerical analysis has been performed considering several aspect ratios $(A=0.2, A=0.25$, $\mathrm{A}=0.3, \mathrm{~A}=0.4, \mathrm{~A}=0.5$ ). The Prandtl number is $\mathrm{Pr}=30$ (corresponding to a silicone oil having a kinematic viscosity $\left.v=2 \cdot 10^{-6}\left[\mathrm{~m}^{2} / \mathrm{s}\right]\right)$.

The presence of buoyancy forces (bridge heated from above or from below) has been introduced in order

to evaluate the influence of gravity effects on the development and characteristics of instability of Marangoni flow. In the numerical simulations the Rayleigh number corresponding to each case studied has been calculated considering a diameter of $0.4 \mathrm{~cm}$ for the disks supporting the liquid zone, $\mathrm{g}=\mathrm{g}_{\mathrm{o}}=9.81$ $\left[\mathrm{m} / \mathrm{s}^{2}\right]$ and a length $\mathrm{L}=\mathrm{D} \cdot \mathrm{A}$.

Different non-uniform grids have been used to adequately meet the special features of the Marangoni flow in the liquid bridge. For high aspect ratios $(A>0.3)$ the computational points have been distributed almost uniformly in the computational domain, whereas for low aspect ratios $(\mathrm{A}<0.3)$ finer grids have been introduced near the free surface to enhance the resolution in the thin Marangoni boundary layer.

For $\mathrm{A}=0.5,24$ points have been collocated in the axial direction; this number has been reduced when reducing the length of the liquid bridge ( 24 for $A=0.5$ and 22 for $A=0.2$ ). In radial direction $24=16+8$ points have been used in the case $\mathrm{A}=0.5$ and $28=14+14$ for $\mathrm{A}=0.2$ (i.e the number of points clustered near the free surface has been increased when the aspect ratio is decreased). In particular in azimuthal direction 20 points have been used for $\mathrm{A} \geq 0.4,30$ for $0.2 \leq \mathrm{A} \leq 0.3$ (40 for $\mathrm{A}=0.2$ and heating from above). 


\subsection{Bridge heated from above}

By employing small dimensions of the floating zone it is possible to reduce on ground the effects associated to buoyancy driven convection in comparison to thermal Marangoni convection.

However it must be pointed out that even for small heated liquid volumes of the length of some millimeters on earth, the Rayleigh number $\left(\mathrm{Ra}=\frac{\mathrm{g} \beta_{\mathrm{T}} \Delta \mathrm{TL}^{3}}{v \alpha}\right)$ can be of the order of $\mathrm{O}\left(10^{3}\right)$ or larger and that the ratio between the Rayleigh number and the Marangoni number $\left(\mathrm{Ma}=\frac{\sigma_{\mathrm{T}} \Delta \mathrm{TL}}{\rho v \alpha}\right)$, which measures the relative importance of buoyancy forces and surface tension imbalance $\left(\mathrm{Ra} / \mathrm{Ma}=\frac{\mathrm{g} \rho \beta_{\mathrm{T}} \mathrm{L}^{2}}{\sigma_{\mathrm{T}}}\right)$, increases quadratically with the characteristic dimension of the liquid zone (L).

In the absence of radial gradients, and if thermocapillary forces are negligible, heating from above should yield a stable linear temperature distribution in the liquid bridge (gravity vector parallel to the density gradient).

If thermocapillary effects are present, buoyancy effects can modify the distribution of the temperature and velocity inside the bridge with respect to the situation of pure Marangoni flow. At the beginning, the case of stable and steady Marangoni flow $\left(\mathrm{Ma}<\mathrm{Ma}_{\mathrm{c}}\right)$ will be examined, in order to study the effect of gravity on the laminar Marangoni convection.

The numerical results show that at the center of the liquid bridge, the velocity in $\mu \mathrm{g}$ is larger than at $1 \mathrm{~g}$. The reason for this behaviour is due to the fact that surface tension and buoyancy forces counteract in a liquid bridge heated from above. When gravity changes from $\mu \mathrm{g}$ to $1 \mathrm{~g}$, the center of the vortex appears to move to the interface of the liquid bridge (the radial depth of the vortex cell is decreased, see Figs. 2). The reason is believed to be that the fluid near the cool disk is slowed down by the buoyancy forces; this effect reduces the radial extension of the convection roll.

The influence of the buoyancy effect can also be seen in the temperature field. In zero g conditions a "cold radially elongated zone" is present near the hot disk, which is created by the return flow that brings cool fluid away from the cold wall along the symmetry axis. The cold liquid is carried toward the hot surface at a rather high position. In normal gravity conditions, since the cold liquid, travelling in the return flow, is slowed down by the buoyancy forces, the temperature of this zone is weakened and consequently the radial temperature gradient near the hot disk is decreased. 
Increasing the Marangoni number, the numerical simulations pointed out that, for each case highlighted, the axisymmetric basic state becomes unstable.

When a temperature difference between the supporting disks equal to the critical one is considered, the flow loses its spatial symmetry and a transient unsteady phase develops until a stable supercritical oscillatory three-dimensional state is reached.

The computations indicated that the thermal and flow field organization in the supercritical state may be extremely complex, depending on the geometrical aspect ratio of the liquid bridge (A=L/D).

Similarly to the model of the deformed torous proposed by Preisser, Schwabe and Sharmann [3] the thermo-fluid-dynamic field in the supercritical state, at a fixed time, can be interpreted as the superposition of a sinusoidal azimuthal disturbance to the axisymmetric field, i.e. $F(r, z, \varphi)=F_{o}(r, z)+$ $\widetilde{\mathrm{F}}(\mathrm{r}, \mathrm{z}) \sin (\mathrm{m} \varphi)$ where the subscript (o) refers to the axisymmetric field, $\mathrm{m}$ is the azimuthal wave number (physically it represents the number of sinusoidal distortions in azimuthal direction). To compare the numerical results with the behaviours predicted by previous stability analyses, the dynamics of the three-dimensional perturbations in the neighbourhood of the bifurcation point was analysed by subtracting the azimuthally averaged field $\left[\mathrm{F}_{\mathrm{O}}(\mathrm{z}, \mathrm{r})\right]$ from the numerically computed three-dimensional time-dependent solution F(z,r, $\varphi, \mathrm{t})$ (average flow + perturbation).

The results, summarized in Table II, show a functional dependence of the azimuthal wave number of the critical mode $(\mathrm{m})$ on the aspect ratio of the liquid bridge. The onset wavenumbers are strictly ordered with respect to A. The critical number is $\mathrm{m}=2$ for $\mathrm{A}$ close to 0.5 , whereas higher values have been found for lower aspect ratios. Different values of $m$ were obtained in well defined ranges of the aspect ratio. For instance, Table II shows: $\mathrm{m}=2$ for $0.3<\mathrm{A} \leq 0.5, \mathrm{~m}=3$ for $0.25 \leq \mathrm{A} \leq 0.3$ and $\mathrm{m}=4$ for $\mathrm{A}=0.2$ (very short liquid bridge).

For $\mathrm{m}=2(0.3<\mathrm{A} \leq 0.5)$, in the generic cross-section orthogonal to the liquid bridge axis $(\mathrm{z}=0.5)$ there are four azimuthal convective cells (Fig.3a) and four thermal spots (two hot and two cold) on the liquid bridge surface. For $\mathrm{m}=3(0.25 \leq \mathrm{A} \leq 0.3)$ and $\mathrm{m}=4(\mathrm{~A}=0.2)$ the vortex cells and the surface spots are six and eight respectively (Figs. $3 \mathrm{~b}$ and $3 \mathrm{c}$ ).

The azimuthal organization of the flow field is characterized by an increasing number of convective cells when the aspect ratio is reduced. Since the azimuthal wave number is related to the aspect ratio, the flow structure of the supercritical state is related directly to the value of $\mathrm{m}$. 
Table III shows the numerical and experimental results related to values of the Marangoni number corresponding to typical supercritical conditions $\left(\mathrm{Ma} \cong 1.5 \mathrm{Ma}_{\mathrm{c}}\right)$.

The dependence law between the critical azimuthal wave number and the geometrical aspect ratio provided by the numerical results for heating from above has been confirmed by the experimental results obtained using the microscale apparatus.

In these experiments the value of the critical azimuthal wave number has been determined by observing the surface of the liquid bridge using a thermocamera. The thermocamera gives the number of the spots on an extension of the free surface of the bridge corresponding to 160 degrees. This number represents the critical azimuthal wave number. In fact, on the entire angular extension of the bridge free surface (360 degrees) there are $2 \mathrm{~m}$ spots ( $\mathrm{m}$ hot and $\mathrm{m}$ cold): if the thermocamera provides the number of the spots on 160 degrees, this number is $\mathrm{m}$.

For $A=0.4$ there are two thermal spots on the bridge surface and hence $\mathrm{m}$ is 2 (Figs. $4 \mathrm{a}-\mathrm{d}$ and $4 \mathrm{e}-\mathrm{h}$ show the surface disturbance temperature distribution determined numerically and experimentally respectively; in these figures the period $\tau$ has been divided into 4 parts and the temperature field is shown in figs a,b,c,d corresponding to $\mathrm{t}=0, \tau / 4, \tau / 2,3 \tau / 4)$; for $\mathrm{A}=0.25$ there are three thermal spots on the bridge surface and hence $\mathrm{m}$ is 3 (Figs. 5a-b and 5c-d show the surface disturbance temperature distribution determined numerically and experimentally respectively; in these figures the period $\tau$ has been divided into 2 parts and the temperature field is shown in figs a,b corresponding to $t=0$, and $t=\tau / 2$ ) in perfect agreement with the numerical computed azimuthal wave numbers. Also the experimental frequencies are in agreement with the numerical computed ones and show a strong increase when the geometrical aspect ratio of the bridge is decreased (Table III).

The numerical results point out moreover that, when heating from above the azimuthal critical wave numbers are almost the same with respect to the $\mathrm{g}=0$ situation (there is a difference only for the case $\mathrm{A}=0.3$ since $\mathrm{m}=2$ for $\mu \mathrm{g}$ conditions and $\mathrm{m}=3$ for $1 \mathrm{~g}$ conditions). This result suggests utilizing liquid bridges heated from above during on ground experiments dedicated to the definition of the experimental set up (number of temperature sensors, thermographic visualization system, etc.) of microgravity experiments (since the flow patterns exhibit similar qualitative behaviours).

However the results reveal that there is a certain increase of the critical Marangoni number and of the critical oscillation frequencies under the action of gravity with respect to the $\mathrm{g}=0$ condition (see Table II showing the non dimensional frequency defined as $\mathrm{f}=\mathrm{fcD}^{2} / \alpha$ where $\mathrm{fc}$ is the computed critical frequency). 
Gravity also produces variations in the frequency spectra. For heating from above, the structure of the oscillations becomes more complicated (Fig. 6) and the oscillatory functions result from the superposition of a second function (with frequency $f_{1}$ ) to the basic fundamental frequency $\left(f_{0}\right)$. Pure Marangoni convection periodic regimes characterized by the existence of a single fundamental frequency turn in quasi-periodic regimes characterized by the superposition of uncommensurable frequencies when gravity effects with heating from above are considered.

The numerical results presented in this paper show moreover that, for all the aspect ratios considered when heating from above, the flow, immediately after the onset, may be properly described by the dynamic model of an "azimuthally standing wave".

The three-dimensional temperature disturbance consists in fact of a number $m$ of couple of spots (hot and cold) "pulsating" at the same azimuthal positions along the interface (minimum and maximum disturbances at fixed azimuthal positions). However, after a certain time, a travelling wave appears characterized by rotating temperature spots along the free surface of the liquid bridge.

This particular behaviour (an azimuthally standing wave that bifurcates in an azimuthally travelling wave after a certain time) is present for all the aspect ratios considered, but from the numerical calculations it can be deduced that the standing wave model is more stable for small aspect ratios (for $0.2<\mathrm{A}<0.3$ the standing wave regime lasts for a long time) and less stable for high aspect ratios $\mathrm{A}>0.3$ (the pulsating regime turns in the rotatory regime after a short transitory).

Also these results are in agreement with the experimental ones. In fact for $A=0.25$ a standing wave regime has been observed (see Figs. 5 with the hot and cold spots pulsating at fixed azimuthal positions) during the duration of the experiment ( 4 minutes) whereas for $A=0.4$ a travelling wave regime (see Figs. 4 with the hot and cold spots rotating in azimuthal direction) has been observed.

The stabilization of the standing wave regimes for low values of the aspect ratio is probably due to the fact that disturbances with higher mode number (low aspect ratio) propagate faster than those with smaller $\mathrm{m}$ (high aspect ratio) and hence have less time to interact and grow and determine the transition from the pulsating regime to the rotating one.

The propagation speed of the disturbances (see Chun and West [2]) is defined as

$$
\widetilde{\mathrm{V}}_{\mathrm{P}}=\frac{2 \pi \mathrm{R}}{\mathrm{m} \tau}
$$

where $\mathrm{R}$ is the radius of the bridge, $\mathrm{m}$ the critical mode number and $\tau$ the period of the oscillations. 
This velocity in non-dimensional form (see Table II) reads:

$$
\mathrm{V}_{\mathrm{P}}=\widetilde{\mathrm{V}}_{\mathrm{P}} \frac{\mathrm{D}}{\alpha}=\frac{2 \pi \mathrm{R}}{\mathrm{m} \tau^{*} \mathrm{D}^{2} / \alpha} \frac{\mathrm{D}}{\alpha}=\frac{\pi}{\mathrm{m}} \mathrm{f}
$$

where $\tau$ and $\mathrm{f}$ are the dimensionless period and the dimensionless frequency of the oscillations respectively.

Table II shows the computed values of Vp; the increase of the propagation speed of the disturbances takes place mainly for $0.3<\mathrm{A}<0.4$ and the numerical and experimental results presented in this paper show that the standing wave regime quickly develops into a travelling one if the aspect ratio is larger than 0.3 .

A possible approach to give a final answer should consist in non linear stability analyses for the solution of the non-linear disturbance amplitude equations after the onset of instability, to study the evolution of the system far from the onset of the instability.

\subsection{Bridge heated from below}

Figs. 7 show the situation of stationary Marangoni flow $\left(\mathrm{Ma}<\mathrm{Ma}_{\mathrm{c}}\right)$, in order to study the effect of aiding buoyancy forces on Marangoni convection.

When the bridge is heated from below and cooled from above, Marangoni and buoyancy flow act in the same direction. The velocity on the free surface increases with respect to heating from above (see the stream-function $\psi$ in Figs. $2 \mathrm{c}-\mathrm{d}$ and $7 \mathrm{a}-\mathrm{b})$ and for $\mathrm{A}<0.3$ other counter-rotating vortex cells induced by buoyancy appear in the interior of the bridge. These additional vortex cells are called "Rayleigh-Benard" convection rolls. The critical Rayleigh number for the occurrence of these phenomena is 1700 (as obtained experimentally by Schwabe et al. [5]) and the Rayleigh numbers corresponding to the conditions considered in the present paper are larger than this value.

Due to the increase of the surface velocity (and of the return flow) and due to the presence of other buoyancy vortex cells in the inner part of the liquid bridge, the temperature field is strongly deformed (see Figs. 7c-f) with respect to the case of heating from above (this behaviour is particularly evident for $\mathrm{A} \leq 0.3)$.

Then the critical Marangoni number for each case considered has been computed and the related supercritical state analysed. The results show that the azimuthal flow organization arising at the onset of instability completely changes according to whether the bridge is heated from above or from below. 
The critical Marangoni numbers are slightly increased (Table IV). The azimuthal wave numbers are slightly different compared with the situation of heating from above (see Figs. 8). Different values of $\mathrm{m}$ were obtained in well defined ranges of the aspect ratio as for heating from above, but for heating from below the ranges corresponding to a fixed value of $\mathrm{m}$ are completely different. For instance, Table II and Table IV show: $\mathrm{m}=4$ for $\mathrm{A}=0.2, \mathrm{~m}=3$ for $\mathrm{A}=0.25$ and $\mathrm{m}=2$ for $0.5<\mathrm{A}<0.3$ for heating from above; when heating from below, the wave number is instead $\mathrm{m}=1$ for $\mathrm{A}>0.3$ (Fig. 8a), $\mathrm{m}=2$ for $\mathrm{A}=0.25$ (Fig. 8b) and $\mathrm{A}=0.3$, and $\mathrm{m}=3$ for $\mathrm{A}=0.2$ (Fig. 8c).

Table $\mathrm{V}$ shows the numerical and experimental results related to values of the Marangoni number corresponding to typical supercritical conditions $\left(\mathrm{Ma} \cong 1.5 \mathrm{Ma}_{\mathrm{c}}\right)$ in the case of bridge heated from below. The dependence law between the critical azimuthal wave number and the geometrical aspect ratio provided by the numerical results is confirmed by the experimental results obtained using the microscale apparatus.

Looking at the temperature spots on the liquid bridge surface provided by the thermocamera in Figs. 9e-h for $A=0.4$ (in Figs. 9 the period $\tau$ has been divided into 4 parts and the velocity field is shown in figs a,b,c,d and e,f,g,h corresponding respectively to $\mathrm{t}=0, \tau / 4, \tau / 2,3 \tau / 4$ for experimental and numerical results) it is clear that the critical wave number is $m=1$ since each spot has an angular extension corresponding to 180 degrees, whereas for $\mathrm{A}=0.25$ (Figs. 10c-d) the critical wave number is $\mathrm{m}=2$ since each spot has an angular extension of 90 degrees.

These experimental results confirm that the azimuthal wave number is shifted to lower values with respect to the situation of heating from above. Moreover, looking at the numerical Fig. 10a-b and the experimental (Fig. 10c-d) for $A=0.25$ it is clear that the instability sets as a standing wave regime while Figs. 9a-d (numerical) and Fig. 9e-h (experimental) show that for $A=0.4$ it is clear that a travelling wave regime is preferred.

For instance, Figs.12 show the temperature oscillations measured by four "numerical probes" positioned at the same axial and radial coordinates but at different azimuthal positions in the case $A=0.5$ (the sketch of the four thermocouples is shown in Fig. 11).

When the bridge is heated from below the phase shift between the temperature profiles is $\pi / 2$ when the two thermocouples are at 90 degrees (Fig.12a) and $\pi$ when the two thermocouples are at 180 degrees (Fig.12b). When the bridge is heated from above the phase shift is $\pi$ when the two considered thermocouples have an azimuthal shift of 90 degrees (Fig.12c) and there is no phase shift when the 
thermocouples are at 180 degrees (Fig.12d). This behaviour can be explained considering that when the bridge is heated from above the critical azimuthal wave number is $m=2$, whereas when the direction of the gravity vector is reversed the critical wave number is $m=1$.

Regardless of the direction of the gravity vector (parallel or antiparallel to the axis) there is an increase of the mode number $\mathrm{m}$ with decreasing of the zone length (decreasing A), but the numerical results point out that for heating from above the product $2 \mathrm{~mA}$ is 1.7 while reversing the direction of the gravity $2 \mathrm{~mA}$ is 1.1 (the constant values that appear in these laws have been computed as average values of the product $2 \mathrm{~mA}$ over the range of aspect ratio investigated $0.2 \leq \mathrm{A} \leq 0.5$; the value const $=1.7$ is caracterized by a maximum uncertainty of $12 \%$ with respect to the minimum or maximum value of $2 \mathrm{~mA}$ whereas in the case of heating from below the uncertainty is $18 \%$ ).

When heating from below the critical oscillation frequencies of the thermofluid-dynamic field always lie below the corresponding values for heating from above (Table II and Table IV). This behaviour is confirmed by the present experimental results that show a decrease of the critical frequency for heating the bridge from below with respect to the heating from above condition.

This finding is in agreement with the experimental results obtained by Velten et al. [4] in the case $\operatorname{Pr}=49$ and in contrast with those obtained by the same authors for $\operatorname{Pr}=7$ and $\operatorname{Pr}=1$ (since in these cases they observed that the critical oscillation frequencies for heating from above lie below the corresponding values for heating from below). Thus from the present results (obtained for $\operatorname{Pr}=30$ ) and those obtained by Velten et al. (for $\operatorname{Pr}=1,7$ and 49) it can be argued that the Prandtl number Pr* corresponding to the "inversion" of the behaviour described above is in the range $7<\operatorname{Pr}^{*}<30$.

The present numerical results point out moreover that the frequency spectra is characterized by the existence of a single fundamental frequency and the behaviour of the oscillations is very regular compared with the heating from above situation .

Also this result is in agreement with the findings of Velten et al. [4] in the case $\mathrm{Pr}=49 . \mathrm{In}_{24} \mathrm{H}_{50}$ zones in fact they did not find transition to aperiodic states in the whole investigated range of aspect ratios and Marangoni numbers.

The present numerical and experimental results reveal that, although the azimuthal structures emerging after the instability are different, the instability mechanism when the combined Marangoni-buoyant flow is considered, is still of a hydrothermal type (i.e. it is related to the mechanism of hydrothermal waves propagation discussed by Kuhlmann et al. [14]) regardless of whether buoyancy effects are aiding or 
opposing to Marangoni flow depending on the relative orientation of the gravity vector and of the temperature gradient. However the standing wave regime is more stable for heating from below with respect to heating from above. On the basis of the interpretation given in section (5.1), this is probably due to the fact that disturbances travel faster when the bridge is heated from below as shown in Table IV. The velocity of propagation of the disturbances is proportional to the ratio of the frequency of the temperature oscillations and of the azimuthal wave number. When the bridge is heated from below, the critical frequency decreases with respect to heating from above as discussed before; however Vp increases due to the fact that the critical wave number is shifted to lower values (the decrease of $\mathrm{m}$ prevails on the decrease of the critical frequency). The increase of $\mathrm{Vp}$ gives rise to a stabilization of the pulsating regimes with respect to the condition of heating from above.

\subsection{Discussion and comparison with other available results}

Hirata, Sakurai et al. [6-7] compared mixed Marangoni-buoyant convection (for bridge heated from above) with the pure thermocapillary flow studying a silicone oil liquid bridge ( $\operatorname{Pr}=30$ and $\mathrm{D}=5 \mathrm{~mm})$ under $1 \mathrm{~g}$ and $\mu \mathrm{g}$. They compared directly experiments performed in $1 \mathrm{~g}$ and $\mu \mathrm{g}$ using a drop shaft tower without changing the experimental setup (the onset of temperature oscillations was observed under $\mu \mathrm{g}$ while a capsule was freefalling and under normal gravity conditions when the capsule was kept at rest).

The critical temperature difference at which the steady state becomes unstable and undergoes a transition to an oscillatory state appeared to be smaller under $\mu \mathrm{g}$ conditions than that under $1 \mathrm{~g}$. Moreover, the fundamental frequency of the temperature oscillations was smaller under $\mu$ g conditions compared to that under $1 \mathrm{~g}$ conditions. The authors observed in many situations a quasi-periodic state with two fundamental frequencies under $1 \mathrm{~g}$ conditions and a periodic state with only one fundamental frequency under $\mu \mathrm{g}$ conditions.

These results are in agreement with the numerical computations reported in this paper. Unfortunately we cannot perform a quantitative comparison because the liquid bridges investigated by Hirata et al. [6-7] have a particularly deformed surface shape due to a non cylindrical volume ( $\left.\mathrm{V} \neq \pi \mathrm{R}^{2} \mathrm{~L}\right)$.

To investigate the influence of buoyancy forces on the stability Velten et al. [4] studied liquid zones heated from above and from below. For relatively large Prandtl number liquids $(\operatorname{Pr}=1,7,49)$ they found that heating from below increases the critical Marangoni number as compared to heating from above. 
According to the present results, their experimental measurements revealed an intriguing behaviour concerning the different influences of buoyancy effects on the critical Marangoni numbers, in liquid bridges heated from below and from above.

The results reported in Figs. 2 could give a first simple explanation for the finding of Hirata et al.[6-7]; in fact, buoyant forces stabilize stationary Marangoni convection in the zone heated from above because thermocapillary forces drive a surface flow of hot lighter fluid downward against buoyancy.

If this were correct, one should expect, in zones heated from below under normal gravity, larger surface flow velocities and correspondingly smaller $\mathrm{Ma}_{\mathrm{c}}$ compared to zones heated from above. On the contrary, the critical Marangoni numbers measured experimentally (Velten et al. [4]) and predicted numerically in this paper are always larger for bridges heated from below than those found for heating from above. This demonstrates that buoyancy stabilizes stationary Marangoni convection not only when thermocapillary and buoyant forces are counteracting but also when these mechanisms are concurrent. A possible explanation for this result is that the onset of the Marangoni instability is related not only to the magnitude of the velocities (because this instability is not caused by inertial effects but it is hydrothermal in nature) but should be explained considering the combined effect of the velocity and temperature fields. It is well known in fact that in the oscillatory regime the time dependence of the Marangoni flow is characterized by small accelerations and decelerations of the primary toroidal convection roll which are caused by minute variations of the surface temperature distribution. The main reason for the instability is the existence of a "cold radially elongated zone" near the hot disk, which is created by the return flow that brings cool fluid away from the cold wall along the symmetry axis. The cold liquid is carried toward the hot surface at a rather high position. This cold zone can generate cold temperature disturbances from inside near the hot free surface and these disturbances can influence the surface temperature distribution giving rise to the instability. In previous results [15] it was pointed out that the instability of Marangoni flow is related to the convective radial heat transport coupled with Marangoni effect. The mechanism of oscillations is based on the temporal interaction between the temperature distribution within the flow field (in particular the "cold radially elongated zone" established inside the liquid bridge near the hot disk as pointed out by Shevtsova et al. [25]) and the temperature sensitive free surface (this is the reason that makes the surface temperature spots appear always near the hot disk). The transition from steady to oscillatory regime occurs when the cold radially elongated zone is able to influence the thermocapillary surface (the sensitivity of the free surface plays a "critical role" in the instability mechanism). 
In this paper it has been shown that heating from above results in lower radial temperature gradients near the hot disk with respect to the case of zero-g conditions whereas heating from below results in larger surface velocities (with respect to the case of heating from above).

In the first case the weak stabilization of the flow with respect to the situation of zero-g conditions can be explained by the reduced sensitivity of the free surface with respect to the case of zero-g conditions; in this case, in fact, the temperature of the cold radially elongated zone is weakened by the buoyancy effects, which slow down the fluid near the cool disk carried in the return flow.

In the latter case (heating from below) the the strong stabilization of the flow with respect to the situation of heating from above is due to the fact that small surface temperature disturbances induced near the hot disk from inside by the cold radially elongated zone have a weaker impact on the accelerations or deceleration of the main surface flow (the sensitivity of the free surface is decreased by the strong surface velocities).

The influence of the buoyancy forces on the radial temperature gradients inside the bridge and on the magnitude of the surface velocity can therefore explain the weak stabilization that occurs when the bridge is heated from above with respect to the situation of zero-g conditions and the strong stabilization that occurs when the bridge is heated from below with respect to the situation of heating from above.

The results of the linear stability analyses of Kuhlmann et al. [21] and Wanschura et al. [22] explain this behaviour in terms of a delicate balance between stabilizing and destabilizing buoyancy effects.

They suppose that since hydrothermal waves are characterized by strong axial vorticity while buoyant convection favors convection rolls with strong horizontal vorticity, therefore both types of convection structures are incompatible in the sense that their respective transport mechanism exclude each other yielding a stabilization of the basic state. Regarding the occurrence of the standing wave regime or of the travelling wave regime, unfortunately the linear stability analyses cannot provide information on this subject since they do not consider the spatio-temporal behaviour of the system.

The only experimental information available are those of Velten et al. [4], those reported in the excellent overview of Frank and Schwabe [8] and those of Shevtsova, Mojahed and Legros [20].

For both heating conditions, several aspect ratios and different Prandtl numbers, Frank and Schwabe [8] describe in detail the structure of pulsating and rotating regimes for the first time through direct observation of the azimuthal flow structure. Comparing the results from three different Prandtl numbers 
$(\operatorname{Pr}=1,7,49)$ and comparing heating from above with heating from below Velten et al. [4] pointed out that the occurrence of rotating regimes is largely decreased in the latter case.

This behaviour can be explained on the basis of the present results. For heating from below the rotating regimes do not disappear; they are simply postponed in time due to the fact that the pulsating regimes are more stable and take a long time to develop in rotating ones.

Velten et al. [4] explain the fact that pulsating regimes (which they call $\mathrm{m}=0$ axially running waves with deformed wave front) are favored for heating from below on the basis of the theory of Xu and Davis [12]. According to this theory the energy source for the axially running waves and for the travelling waves should be the radial temperature gradient and the radial velocity gradient respectively. Velten et al. [4] attribute the decrease of the observed rotating regimes in the case of heating from below to the reduction of the radial velocity gradient and to the increase of the radial temperature gradient due to the buoyancy effects.

This theory however, cannot be accepted on the basis of the present results. The pulsating regime in fact is not a "pure" axially running wave with $\mathrm{m}=0$ and therefore it does not correspond to the prediction of $\mathrm{Xu}$ and Davis [12] obtained in the case of infinite liquid bridges.

Regarding the transition between the pulsating regime and the travelling regime that has been observed in the present results for all the aspect ratios considered, very few information is available in literature. Velten et al. [4] in many cases found unexpected behaviors (that they were not able to explain) that could be interpreted as transition between pulsating and rotating regimes.

Using three thermocouples having the same axial and radial coordinates but different azimuthal positions, they measured in many situations phase shifts changing continuously in time.

When the regime is pulsating (surface temperature spots pulsating at fixed azimuthal positions) and two thermocouples are located on the same spot, they give temperature signals that do not show phase difference; when the regime becomes rotating the two thermocouples give signals that show a constant phase shift related to the angular distance between them. Between these two regimes a hybrid regime exists that should be called "pulso-rotating"; during this regime the phase shift between the two thermocouples increases in time starting from zero until it reaches a constant value corresponding to the rotating regime; during this phase the phase shift corresponds to values of the azimuthal wave number that are not entire as observed by Velten et al. [4]. 
Transition from a standing wave regime to travelling wave regime has been observed however directly by Monti, Savino, Lappa and Fortezza [19] in the case of a liquid bridge of silicone oil $(\mathrm{Pr}=45)$ and aspect ratio $A=0.7(m=1)$, and by Shevtsova, Mojahed and Legros [20] in the case of $\operatorname{Pr}=105$ and $A=0.6(m=1)$. These authors used four thermocouples located at the same axial and radial coordinates but at different azimuthal positions to "detect" the transition from the pulsating regime to the rotating one.

\section{Conclusions}

The interaction of buoyancy and Marangoni flow in a vertical cylindrical liquid bridge with an adiabatic free lateral surface and isothermal top and bottom walls has been studied numerically in a transient threedimensional simulation and experimentally using a micoscale facility.

Most of the existing information in literature for high Prandtl numbers $\left(\mathrm{O}\left(10-10^{2}\right)\right)$ comes from experiments and from linear stability analysis based on a linearized form of the equations that does not permit any information to be obtained on the spatio-temporal behaviour of the liquid bridge in the supercritical state. In the present paper a systematic and parametric analysis of the features of the mixed buoyancy-Marangoni instability for a high Prandtl liquid $(\mathrm{Pr}=30)$ is performed through full 3D solution of the time-dependent, non linear and complete Navier Stokes equations.

The spatio-temporal behaviour of the supercritical regime has been studied in detail (no numerical works are available in literature on this subject and linear stability analyses are unable to do this).

The influence of buoyancy effects on the flow instability and in particular on the critical wave number (m) and on the spatio-temporal structures (pulsating or rotating) has been considered.

The results show that the azimuthal flow organization arising at the onset of instability completely changes according to whether the bridge is heated from above or from below. In particular, when the liquid bridge is heated from below, the critical Marangoni number is larger and the critical wave number ( $\mathrm{m}$ that represents the number of sinusoidal distortions in azimuthal direction) is smaller compared with the case of the bridge heated from above.

These results show that gravity effects may have a large effect on Marangoni flow instability when the bridge is heated from below, even if the experiments are performed reducing the typical length of the liquid bridge to a few millimeters (microscale experimentation).

For the dimensionless wave number of the instability two correlation functions are found: $2 \mathrm{~mA}=1.7$ when heating from above and $2 \mathrm{~mA}=1.1$ when heating from below. 
For the first time the influence of the gravity field on the transition from standing wave regimes to travelling wave regimes has been studied. The results indicate that the mechanism of the instability is of a hydrothermal type and characterized by a transition from a pulsating regime to a rotating regime. This mechansim is present for both heating conditions, but for heating from below the standing wave regime is more stable.

The weak stabilization that occurs when the bridge is heated from above with respect to the situation of zero-g conditions and the strong stabilization that occurs when the bridge is heated from below with respect to the situation of heating from above have been explained in terms of the influence of the buoyancy forces on the radial temperature gradients inside the bridge and on the magnitude of the surface velocity.

For the first time the influence of the gravity field on the fourier spectra of the supercritical state has been examined using numerical simulation.

\section{Acknowledgments}

This work is part of the PhD thesis of M.Lappa.

The authors would like to thank the Italian Aerospace Research Center (CIRA) which allowed the numerical calculations on the Silicon Graphics Power Challenge Supercomputer to be made.

\section{References}

[1] D. Schwabe, A. Scharmann A., F: Preisser, R. Oeder, "Experiments on surface tension driven flow in floating zone melting", J. Crystal Growth, 43, 305, (1978)

[2] C.H. Chun, W. West, "Experiments on the transition from the steady to the oscillatory Marangoni convection of a floating zone under reduced gravity effect", Acta Astronautica 6, 1073 (1979).

[3] F. Preisser, D. Schwabe, A. Scharmann, "Steady and oscillatory thermo capillary convection il liquid columns with free cylindrical surface", J.Fluid Mech. 126, 545 (1983).

[4] R. Velten, D. Schwabe, A. Scharmann, "The periodic instability of thermocapillary convection in cylindrical liquid bridges", Phys.Fluids A 3, 267 (1991).

[5] D. Schwabe , P. Hintz, S. Frank, "New Features of Thermocapillary Convection in Floating Zones Revealed by Tracer Particle Accumulation Structures", Microgravity sci. technol. 9, 163 (1996). 
[6] A. Hirata, M. Sakurai, N. Ohishi, "Effect of gravity on Marangoni convection in a liquid bridge", J.Jpn. Soc. Microgravity Appl., 14, 130, (1997)

[7] A. A. Hirata, M. Sakurai, N. Ohishi, M. Koyama, T. Morita, H. Kawasaki, "Transition process from laminar to oscillatory Marangoni convection in a liquid bridge under normal and microgravity", J.Jpn. Soc. Microgravity Appl., 14, 137, (1997)

[8] S. Frank, D. Schwabe, "Temporal and spatial elements of thermocapillary convection in floating zones", Experiments in Fluids, 23, 234 (1998).

[9] R. Monti and R. Fortezza, "Non-intrusive techniques for thermal measurements in microgravity fluid science experiments", Adv. Space Res., 6, 69, (1986)

[10] R. Monti et al., "First Results from 'Onset' Experiment during Spacelab Mission D-2", Proceedings of the Norderney Symposium on Scientific Results of the German Spacelab Mission D2, Editors P.R. Sahm, M.H. Keller, B. Schiewe, 247 (1995).

[11] K.A. Muehlner , M. F. Schatz , V. Petrov , W.D. McCormick , J.B. Swift , H.L. Swinney, "Observation of helical traveling-wave convection in a liquid bridge", Phys. Fluids 9 (6), 1850 (1997).

[12] J.J. Xu. and S.H. Davis, "Convective thermocapillary instabilities in liquid bridges", Phys. Fluids 27, 1102 (1984).

[13] G.P. Neitzel., K.T. Chang, D.F. Jancowski, H.D. Mittelmann, "Linear stability of thermocapillary convection in a model of the float-zone crystal-growth process", Phys. Fluids A 5, 108 (1992).

[14] H.C. Kuhlmann, H.J. Rath, "Hydrodinamic instabilities in cylindrical thermocapillary liquid bridges", J.Fluid Mech. , 247 , 247 (1993).

[15] M. Wanschura, V. Shevtsova, H.C. Kuhlmann, H.J. Rath, "Convective instability mechanism in thermocapillary liquid bridges", Phys. Fluids A 5, 912 (1995).

[16] R. Rupp, G. Muller, G. Neumann, "Three dimensional time dependent modelling of the Marangoni convection in zone melting configurations for GaAs", Journal of Crystal growth 97, 34 (1989).

[17] R. Savino and R. Monti, "Oscillatory Marangoni convection in cylindrical liquid bridges", Phys. Fluids, 8, 2906 (1996)

[18] R. Monti, R. Savino, M. Lappa, "Oscillatory Thermocapillary flows in simulated floating zones with time-dependent boundary conditions"; Acta Astronautica 41, 863, (1997).

[19] R. Monti, R. Savino, M. Lappa and R. Fortezza, "Scientific and Technological Aspects of a Sounding Rocket Experiment on Oscillatory Marangoni Flow", Space Forum, 2, 293 (1998). 
[20] V. Shevtsova, M. Mojahed, J.C. Legros, "The loss of stability in ground based experiments in liquid bridges", 49th Congress of the International Astronautical Federation, Melbourne, Australia, Sept.28Oct. 21998

[21] H.C. Kuhlmann, M. Wanschura, J. Leypoldt, H.J. Rath, "Buoyant-thermocapillary convection and three-dimensional flow in liquid bridges: comparison of numerical and experimental results", Microgravity Q., 6, 65, (1996)

[22] M. Wanschura, H.C. Kuhlmann, H.J. Rath, "Linear stability of two-dimensional combined buoyantthermocapillary flow in cylindrical liquid bridges", Physical Review E, 55 (6), 7036, (1997)

[23] M. Lappa , R. Savino, "Parallel solution of three-dimensional Marangoni flow in liquid bridges", Int. J. Num. Meth. Fluids, 31, 911, (1999)

[24] C.A.J. Fletcher, "Computational techniques for fluid-dynamics", (Springer Verlag, Berlin, 1991)

[25] V. Shevtsova, J.C. Legros, "Influence of the free surface shape on stability of liquid bridges", Joint Xth European and VIth Russian Symposium on Physical Sciences in Microgravity, St. Petersburg, Russia, 15-21 June 1997

\begin{tabular}{|c|c|}
\hline Grid size & Minimum stream function \\
\hline Kuhlmann and Rath (1993) & -4.1 \\
\hline Wanschura et al.(1995) $25 \times 14$ & -4.08 \\
\hline Wanschura et al.(1995) $50 \times 25$ & -4.10 \\
\hline Present results $20 \times 20$ & -4.03 \\
\hline Present results $24 \times 24$ & -4.075 \\
\hline Present results $30 \times 30$ & -4.094 \\
\hline Present results $40 \times 40$ & -4.10 \\
\hline Present results $50 \times 50$ & -4.103 \\
\hline
\end{tabular}

\section{Table Ia}

Minimum stream function of the axisymmetric flow as a function of mesh spacing in the case of uniform grids $(\mathrm{A}=0.5, \operatorname{Pr}=10, \mathrm{Ma}=1000)$ 


\begin{tabular}{||l|c||}
\hline Grid size & Minimum stream function \\
\hline \hline Present results 24 x 24 & -4.075 \\
\hline Present results 24 x (20+4) & -4.077 \\
\hline Present results 24 x (18+6) & -4.085 \\
\hline Present results 24 x (16+8) & -4.10 \\
\hline Present results 24 x (14+10) & -4.102 \\
\hline
\end{tabular}

\section{Table Ib}

Minimum stream function of the axisymmetric flow as a function of mesh spacing in the case of non uniform grids $(\mathrm{A}=0.5, \mathrm{Pr}=10, \mathrm{Ma}=1000)$.

The number of points clustered near the free surface (using the stretching function (s) due to Roberts and Eiseman) are specified using the notation $\mathrm{Nz} \times\left(\mathrm{Nr}_{\mathrm{b}}+\mathrm{Nr}_{\mathrm{s}}\right)$ (where $\mathrm{Nr}_{\mathrm{s}}$ is the number of points stretched near the free surface and $\mathrm{Nr}_{b}$ the points uniformly distributed in the bulk over a radius $\mathrm{R}_{b}=0.7 \mathrm{R}$ )

\begin{tabular}{|l|c|c|c||}
\hline \hline Grid size & Vz at $\mathrm{r}=0.25$ & Vz at $\mathrm{r}=0.5$ & Vz at $\mathrm{r}=1.0$ \\
\hline \hline $20 \times 20$ & 14.665 & 16.60 & -43.83 \\
\hline $24 \times 24$ & 14.59 & 16.624 & -43.82 \\
\hline $30 \times 30$ & 14.504 & 16.622 & -43.80 \\
\hline $40 \times 40$ & 14.44 & 16.605 & -43.77 \\
\hline $24 \times(16+8)$ & 14.446 & 16.58 & -43.79 \\
\hline
\end{tabular}

Table Ic

Axial velocity of the axisymmetric flow at $\mathrm{z}=0.5$ and at different radial positions as a function of grid spacing $(\mathrm{A}=0.5, \mathrm{Pr}=10, \mathrm{Ma}=1000)$

\begin{tabular}{||l|l||}
\hline Grid size & $\mathrm{f}$ \\
\hline $24 \times(16+8) \times 20$ & 75.47 \\
\hline $24 \times(16+8) \times 25$ & 78.4 \\
\hline $24 \times(16+8) \times 30$ & 80 \\
\hline
\end{tabular}

Table Id

$3 \mathrm{D}$ grid refinement study for $\mathrm{A}=0.5, \mathrm{Pr}=4, \mathrm{Ma}=4800$

(the frequency is posed in non-dimensional form using. $\mathrm{D}^{2} / \alpha$ as reference time) 


\begin{tabular}{|c|c|c|c|c|c||c|c||}
\hline \hline $\mathrm{A}$ & $\mathrm{Ra}$ & $\mathrm{Ma}_{\mathrm{c}}$ & $\mathrm{m}$ & $\mathrm{f} \cdot 10^{-2}$ & $\mathrm{Vp} \cdot 10^{-2}$ & $\begin{array}{c}\mathrm{m} \\
\text { (zero g) }\end{array}$ & $\begin{array}{c}\mathrm{f} \cdot 10^{-2} \\
\text { (zero g) }\end{array}$ \\
\hline \hline 0.2 & $-2.910^{3}$ & $2.510^{4}$ & 4 & 11.82 & 9.27 & 4 & 11.36 \\
\hline 0.25 & $-4.4210^{3}$ & $2.4510^{4}$ & 3 & 7.79 & 8.16 & 3 & 7.38 \\
\hline 0.3 & $-6.1710^{3}$ & $2.410^{4}$ & 3 & 5.91 & 6.18 & 2 & 5.17 \\
\hline 0.4 & $-1.0910^{4}$ & $2.3510^{4}$ & 2 & 3.55 & 5.57 & 2 & 3.08 \\
\hline 0.5 & $-1.6210^{4}$ & $2.2510^{4}$ & 2 & 2.72 & 4.26 & 2 & 2.77 \\
\hline
\end{tabular}

Table II: Influence of the aspect ratio on the critical parameters (numerical results, bridge heated from above)

\begin{tabular}{||c|c|c|c|c|c|c|c|c||}
\hline \hline $\mathrm{A}$ & $\mathrm{Ra}$ & $\mathrm{Ma}$ & $\begin{array}{c}\mathrm{m} \\
\text { (num.) }\end{array}$ & $\begin{array}{c}\mathrm{f} \cdot 10^{-2} \\
\text { (num.) }\end{array}$ & $\begin{array}{c}\mathrm{Vp} \cdot 10^{-2} \\
\text { (num.) }\end{array}$ & $\begin{array}{c}\mathrm{m} \\
(\mathrm{exp} .)\end{array}$ & $\begin{array}{c}\mathrm{f} \cdot 10^{-2} \\
(\text { exp. })\end{array}$ & $\begin{array}{c}\mathrm{Vp} \cdot 10^{-2} \\
\text { (exp.) }\end{array}$ \\
\hline \hline 0.25 & $-7.210^{3}$ & $3.7510^{4}$ & 3 & 8.72 & 9.13 & 3 & 8.85 & 9.26 \\
\hline 0.4 & $-1.6710^{4}$ & $3.610^{4}$ & 2 & 3.40 & 5.34 & 2 & 5.4 & 8.4 \\
\hline 0.5 & $-2.5210^{4}$ & $3.510^{4}$ & 2 & 2.81 & 4.41 & 2 & 4.1 & 6.4 \\
\hline
\end{tabular}

Table III: numerical and experimental results (bridge heated from above)

\begin{tabular}{||c|c|c|c|c|c||}
\hline \hline $\mathrm{A}$ & $\mathrm{Ra}$ & $\mathrm{Ma}_{\mathrm{c}}$ & $\mathrm{m}$ & $\mathrm{f} \cdot 10^{-2}$ & $\mathrm{Vp} \cdot 10^{-2}$ \\
\hline \hline 0.2 & $3.810^{3}$ & $3.310^{4}$ & 3 & 12.3 & 12.87 \\
\hline 0.25 & $5.410^{3}$ & $3.010^{4}$ & 2 & 7.03 & 11.04 \\
\hline 0.3 & $7.2310^{3}$ & $2.810^{4}$ & 2 & 5.38 & 8.45 \\
\hline 0.4 & $1.1810^{4}$ & $2.5510^{4}$ & 1 & 2.86 & 8.98 \\
\hline 0.5 & $1.7310^{4}$ & $2.410^{4}$ & 1 & 2.15 & 6.76 \\
\hline
\end{tabular}

Table IV: Influence of the aspect ratio on the critical parameters (numerical results, bridge heated from below)

\begin{tabular}{||c|c|c|c|c|c|c|c|c||}
\hline $\mathrm{A}$ & $\mathrm{Ra}$ & $\mathrm{Ma}$ & $\begin{array}{c}\mathrm{m} \\
\text { (num.) }\end{array}$ & $\begin{array}{c}\mathrm{f} \cdot 10^{-2} \\
\text { (num.) }\end{array}$ & $\begin{array}{c}\mathrm{Vp} \cdot 10^{-2} \\
\text { (num.) }\end{array}$ & $\begin{array}{c}\mathrm{m} \\
(\text { exp.) }\end{array}$ & $\begin{array}{c}\mathrm{f} \cdot 10^{-2} \\
\text { (exp.) }\end{array}$ & $\begin{array}{c}\mathrm{Vp} \cdot 10^{-2} \\
\text { (exp.) }\end{array}$ \\
\hline \hline 0.25 & $7.6510^{3}$ & $4.210^{4}$ & 2 & 7.35 & 11.54 & 2 & 8.6 & 13.5 \\
\hline 0.4 & $2.0310^{4}$ & $4.310^{4}$ & 1 & 3.19 & 10 & 1 & 4.9 & 15.3 \\
\hline 0.5 & $3.110^{4}$ & $4.210^{4}$ & 1 & 2.50 & 7.85 & 1 & 3.4 & 10.6 \\
\hline
\end{tabular}

Table V: numerical and experimental results (bridge heated from below) 


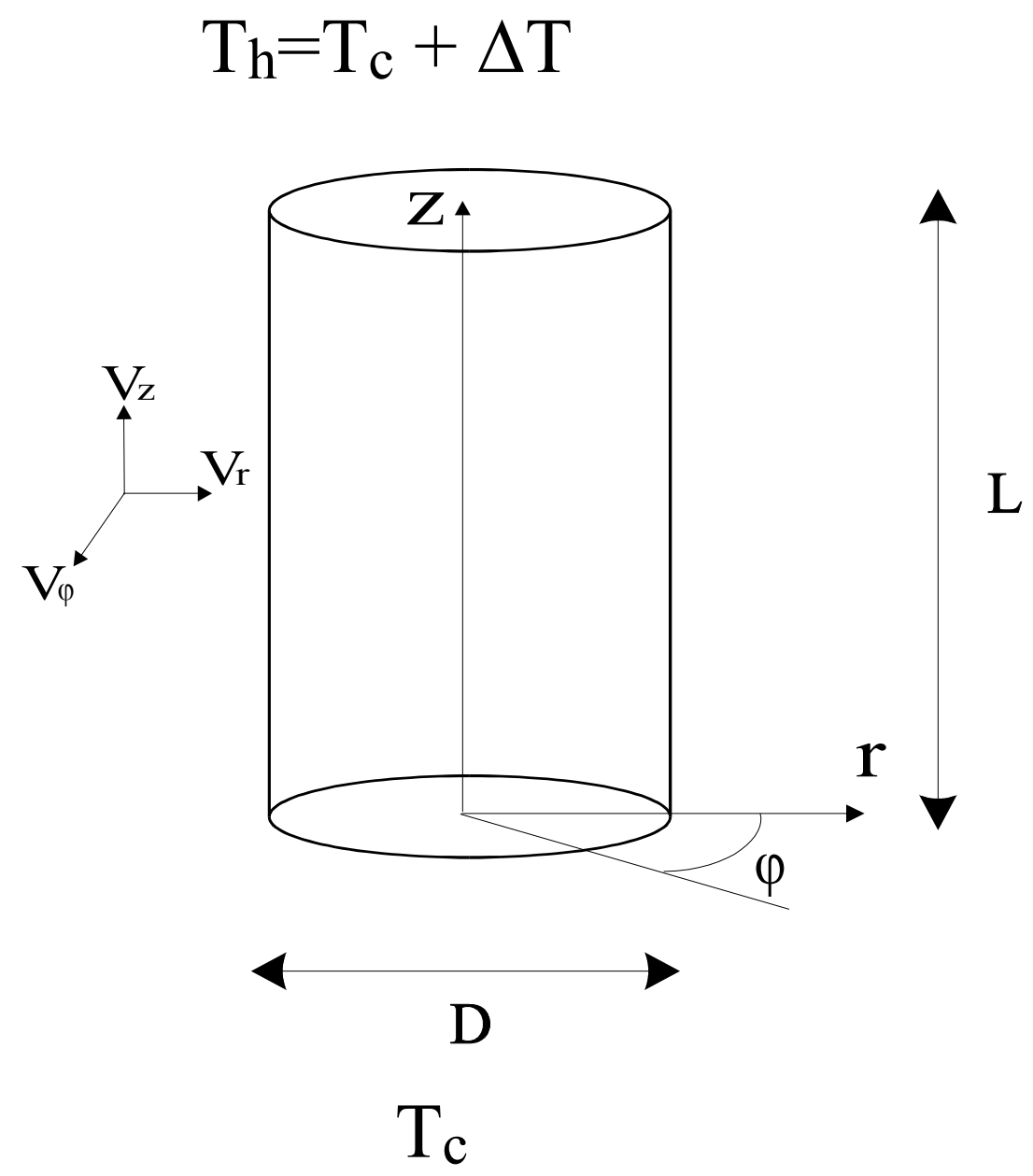

Fig.1: sketch of the liquid bridge 


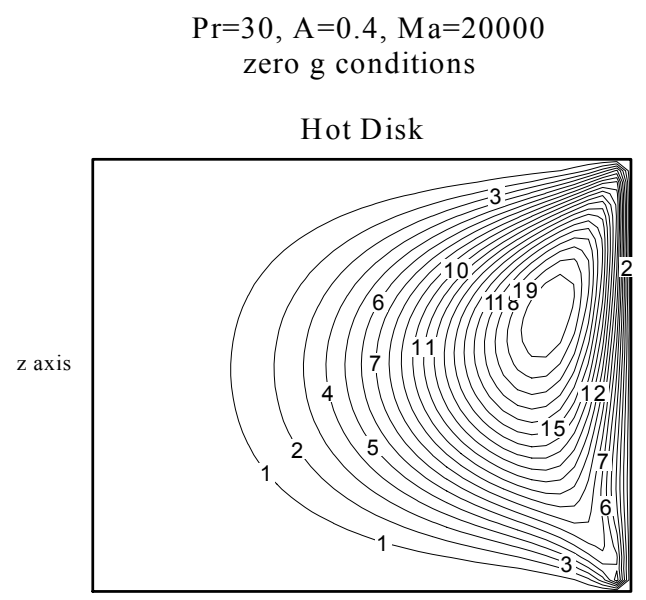

Cold Disk

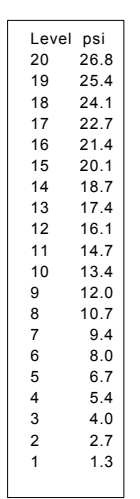

2a)
$\operatorname{Pr}=30, \mathrm{~A}=0.4, \mathrm{Ma}=20000$ heated from above

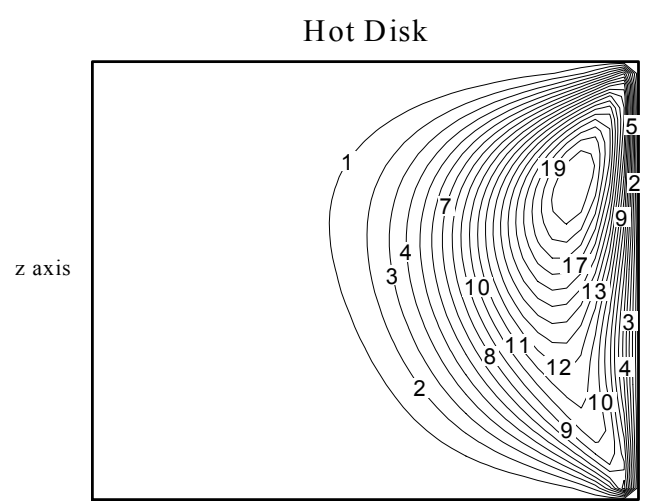

Cold Disk

3a)

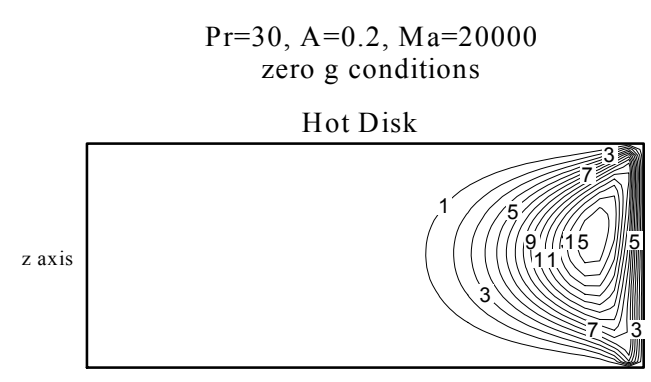

Cold Disk

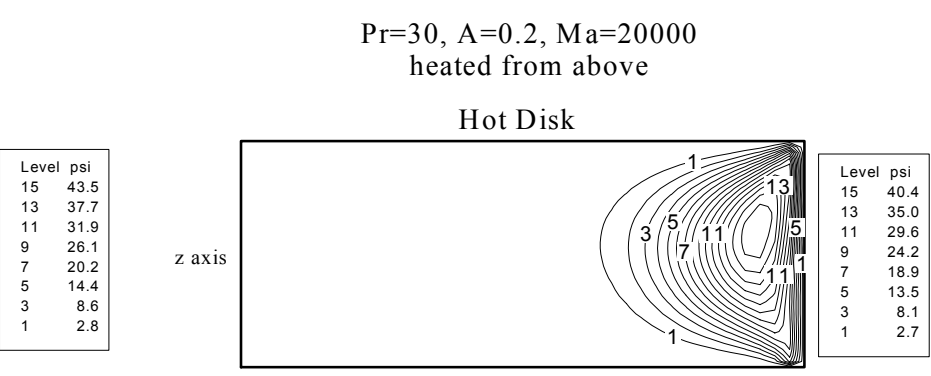

2b)
Cold Disk 

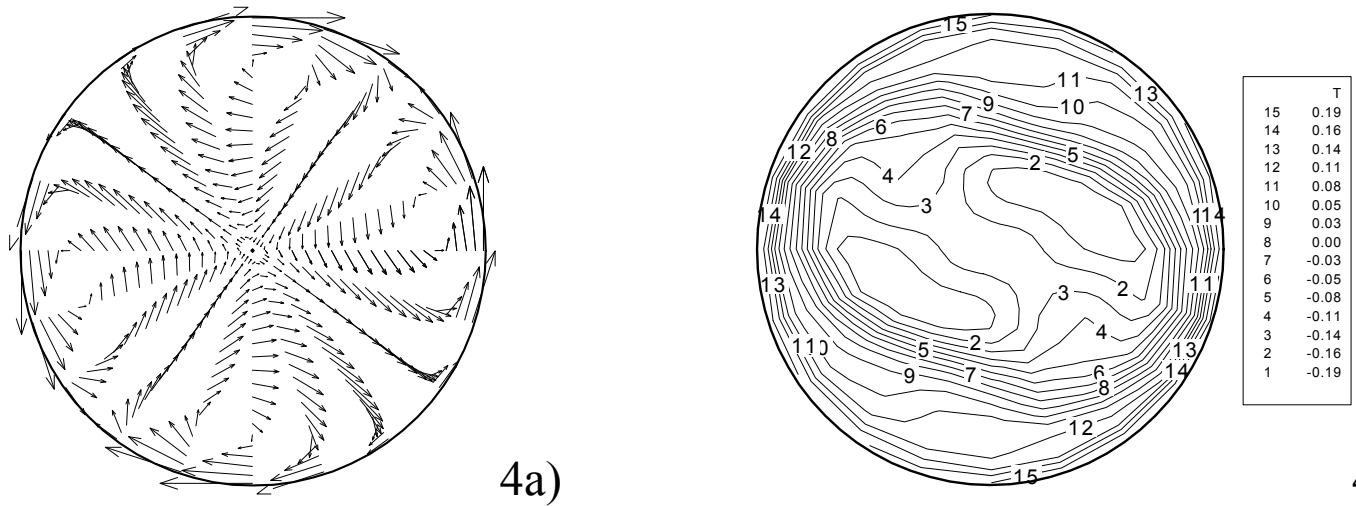

4b)
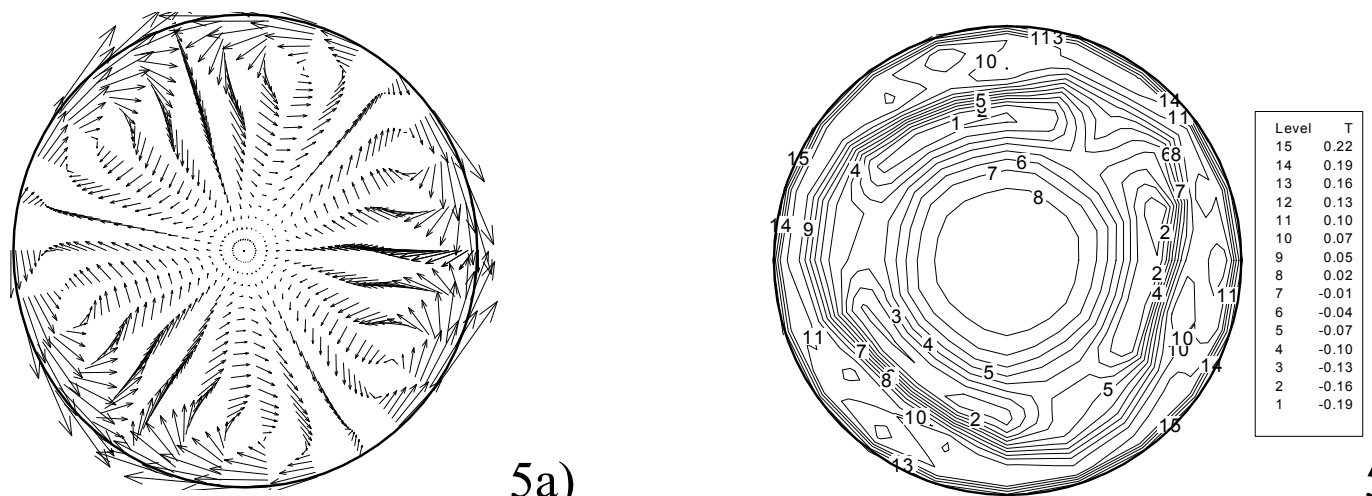

$5 b)$
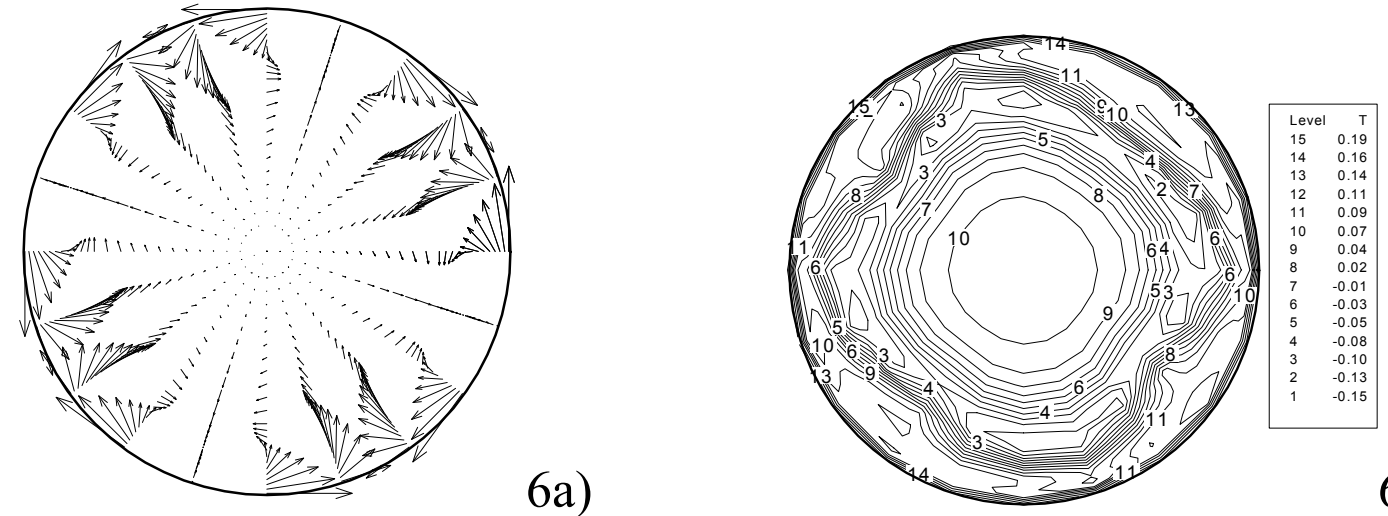

6b)

Numerical results

Velocity field in the section $\mathrm{z}=0.5$ and temperature distribution in the section $\mathrm{z}=0.75$

(bridge heated from above) 


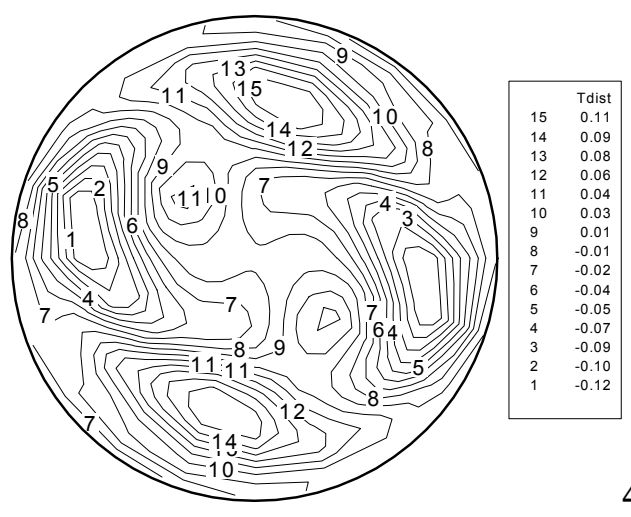

$4 c)$

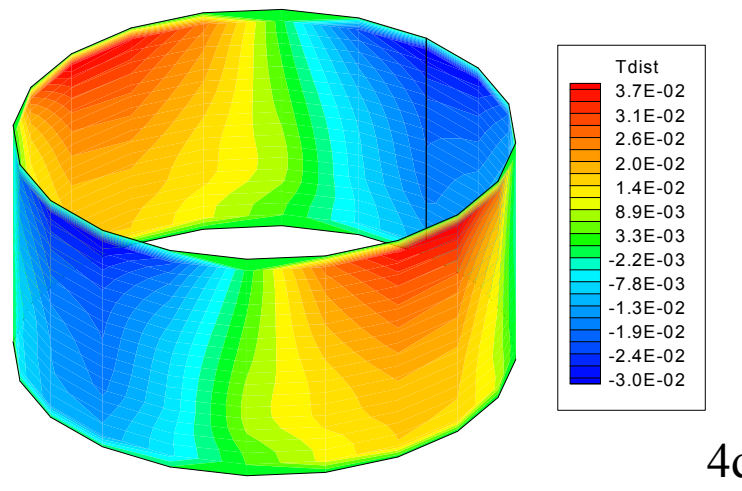

4d)
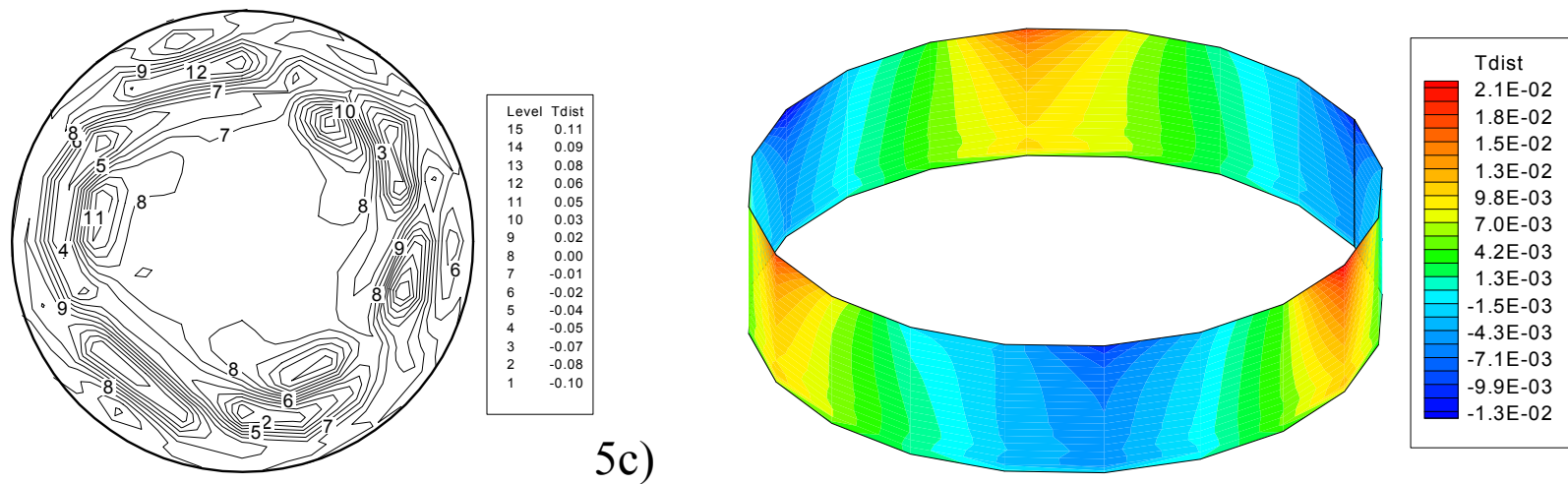

$5 c)$
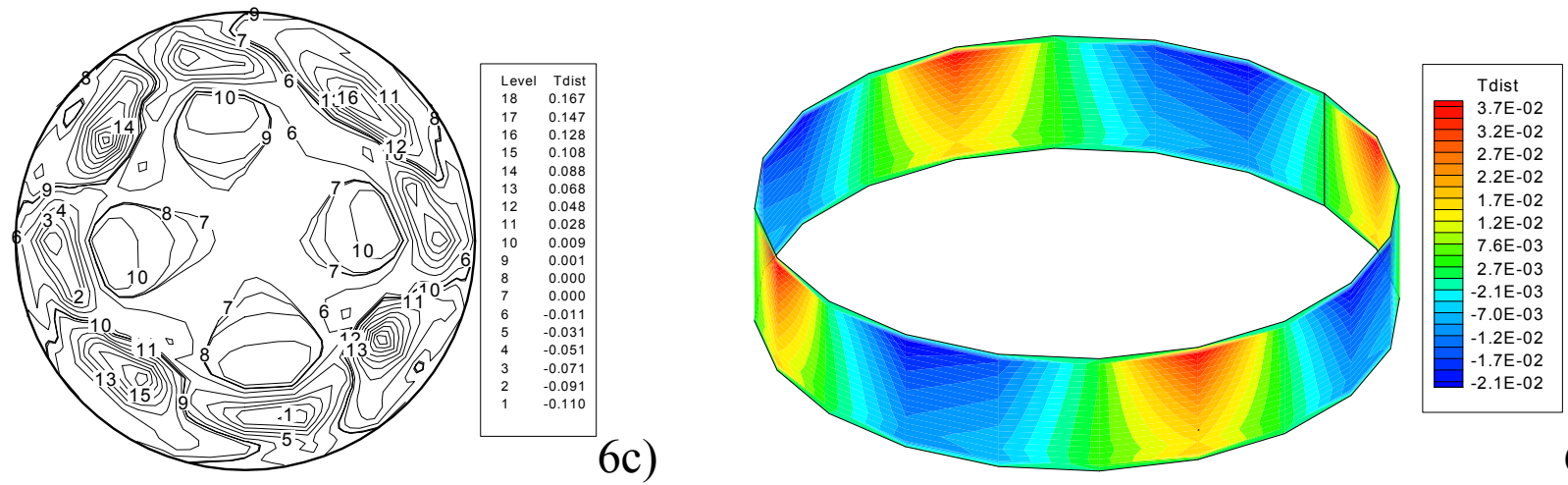

6d)

Temperature disturbances in the section $\mathrm{z}=0.75$ and on the liquid bridge surface (bridge heated from above) 


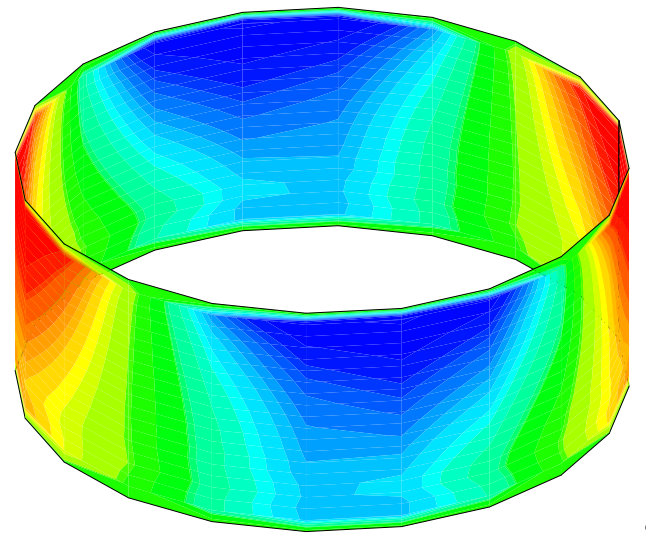

a)
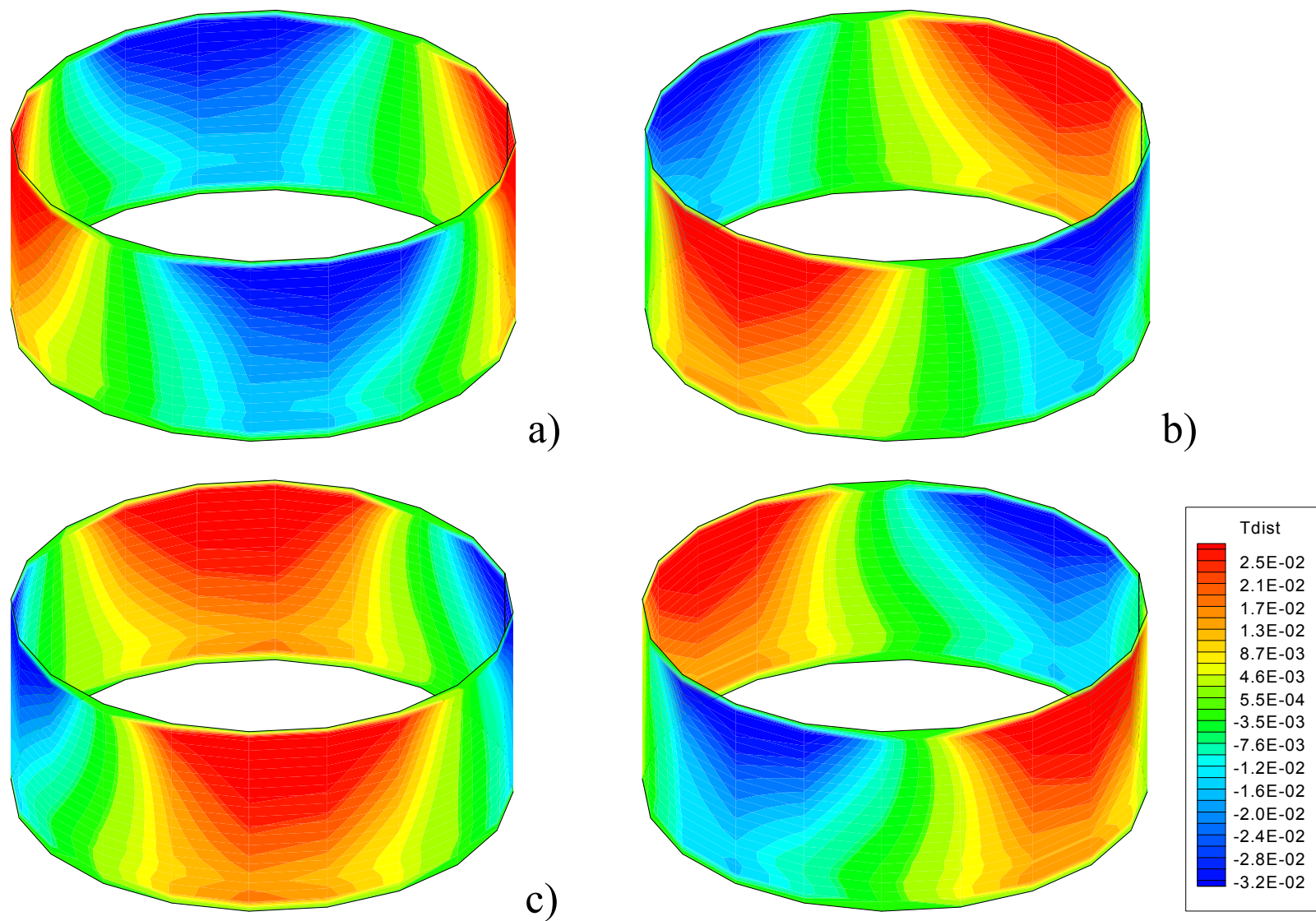

d)

Figs. 7: Temperature disturbance on the brige surface for $A=0.4$ and traveling wave regime (numerical results)

Direction of propagation $\rightarrow$

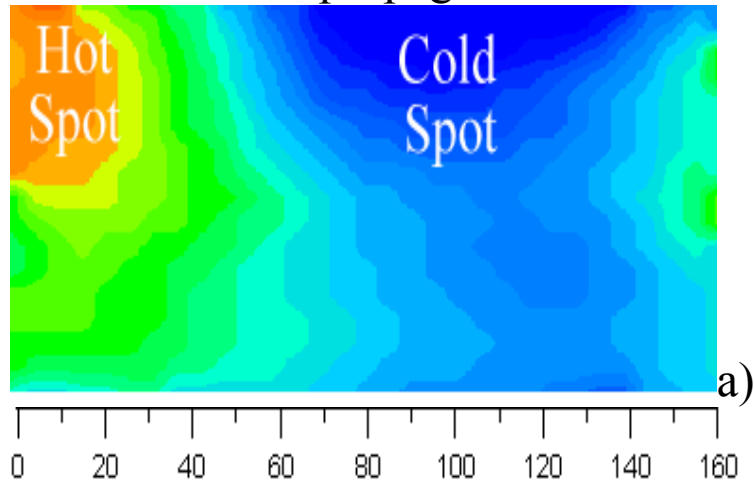

Direction of propagation $\rightarrow$

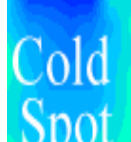

Hot

Spot
Direction of propagation $\rightarrow$

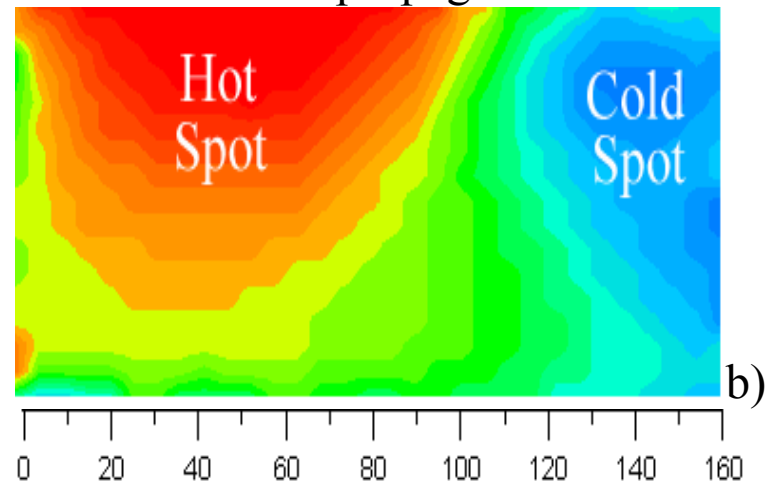

Direction of propagation $\rightarrow$

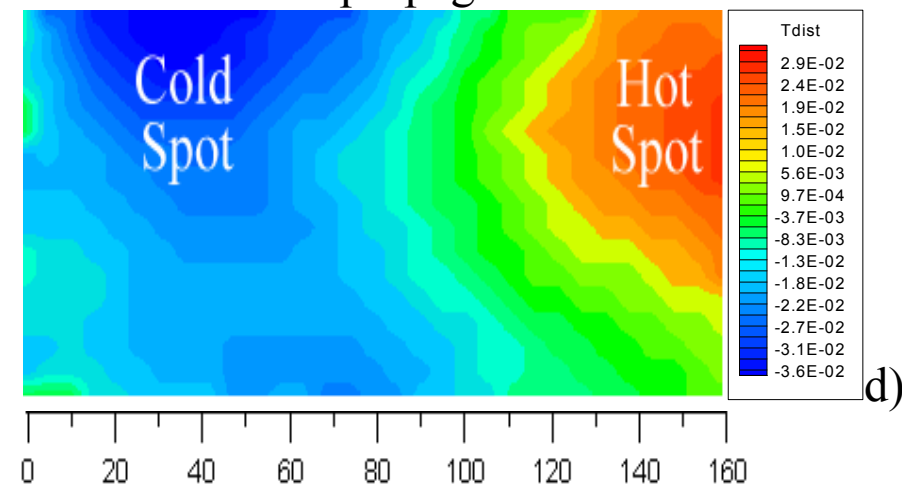

Figs. 8: Temperature disturbance on the brige surface for $A=0.4$ and traveling wave regime (experimental results) 

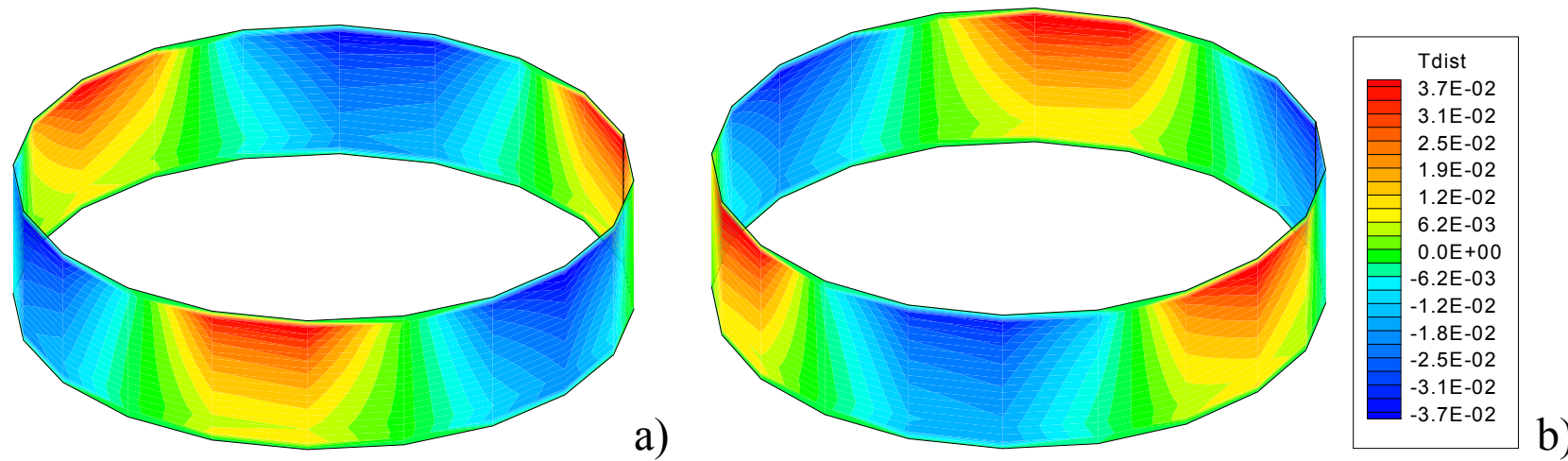

Figs. 9: Temperature disturbance on the brige surface for $A=0.25$ and standing wave regime (numerical results)
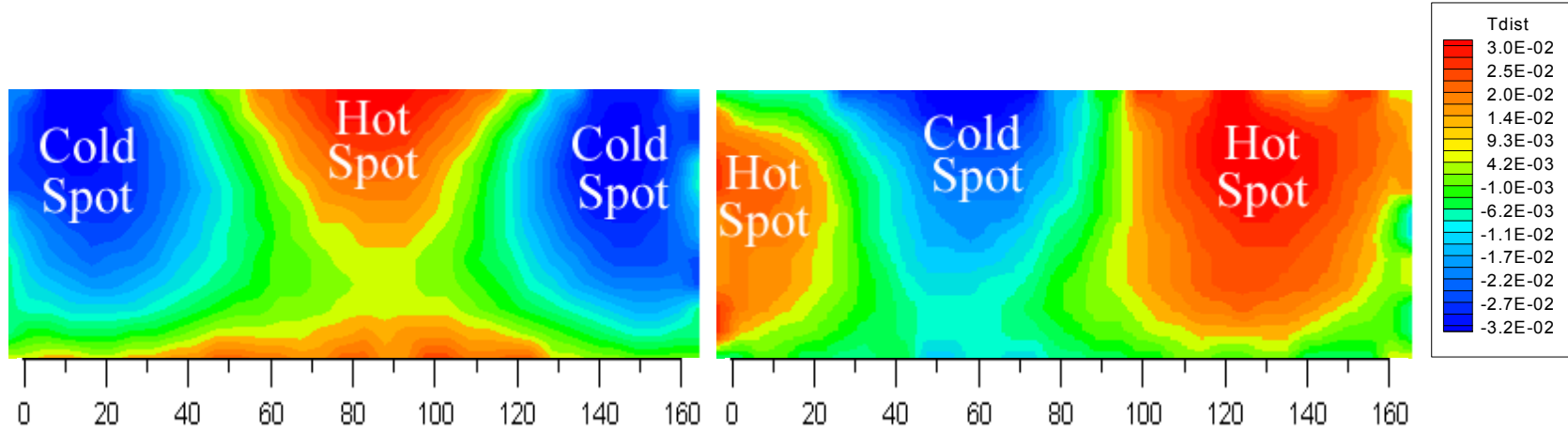

(a)

(b)

Figs. 10: Temperature disturbance on the brige surface for $A=0.25$ and standing wave regime (experimental results) 


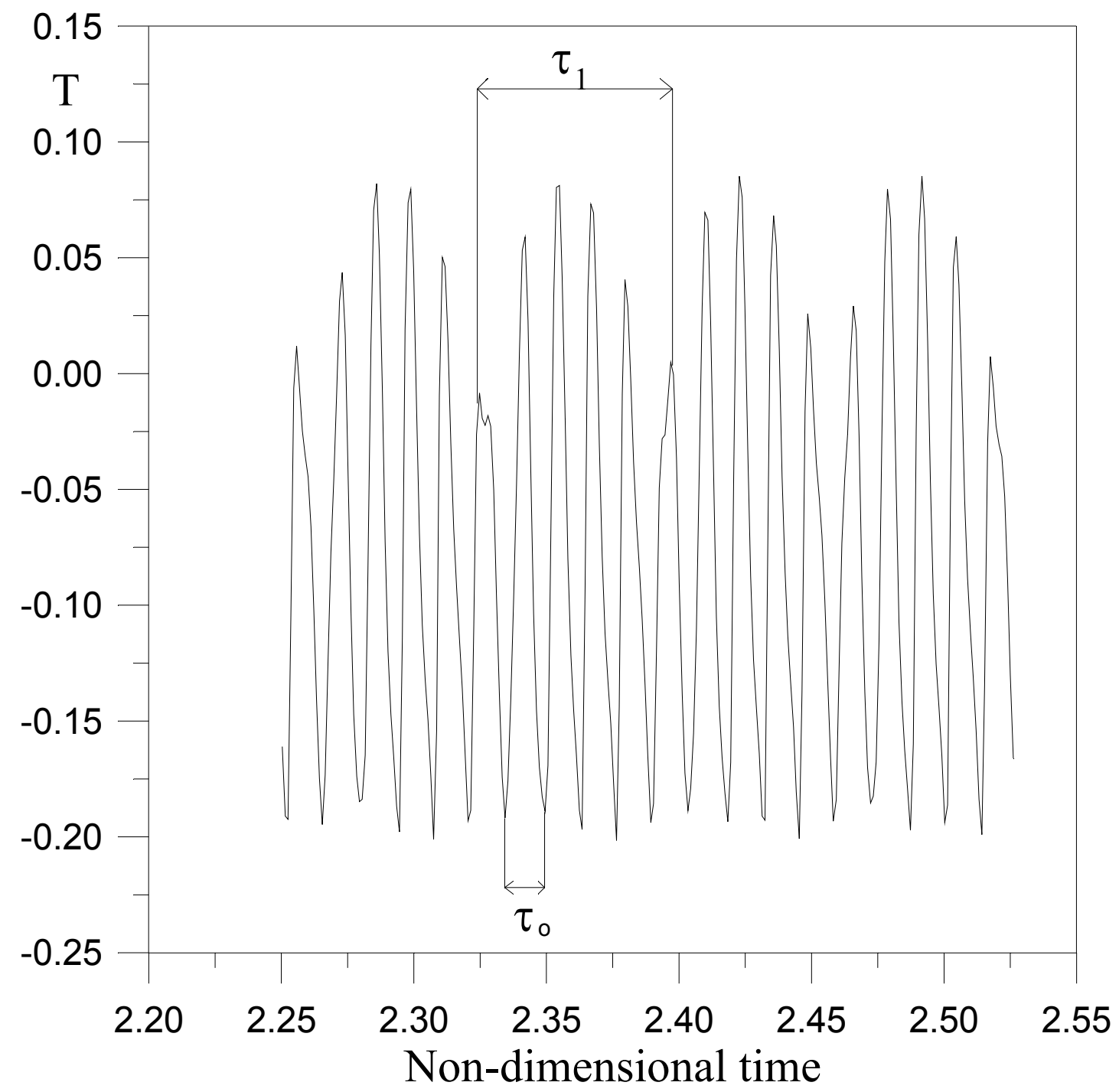

Fig. 11

Temperature oscillation in the point $\mathrm{z}=0.75, \mathrm{r}=0.5, \varphi=0$ $\mathrm{A}=0.5, \mathrm{Ma}=3.5510^{4}$, bridge heated from above 


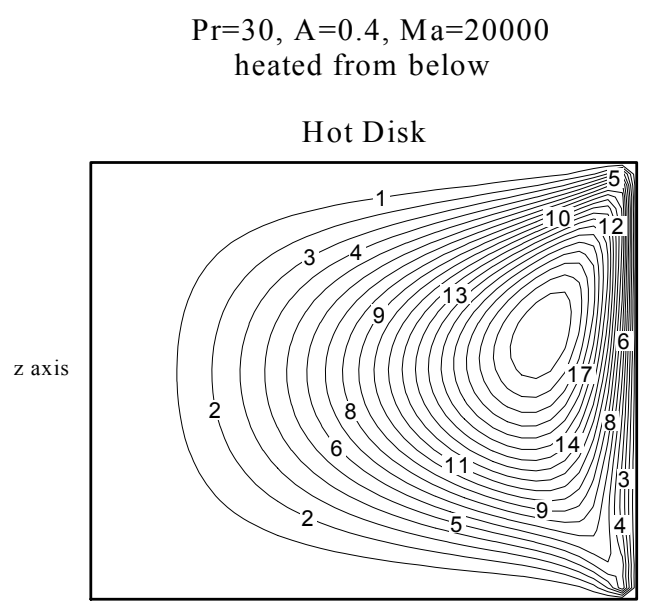

Cold Disk

$\operatorname{Pr}=30, \mathrm{~A}=0.4, \mathrm{Ma}=20000$ heated from below

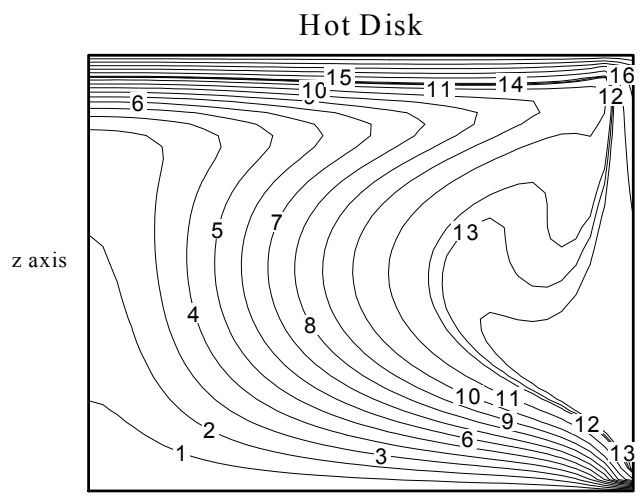

Cold Disk

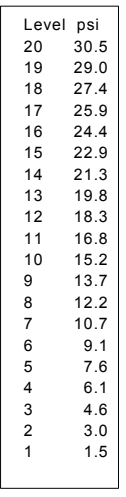

12a)

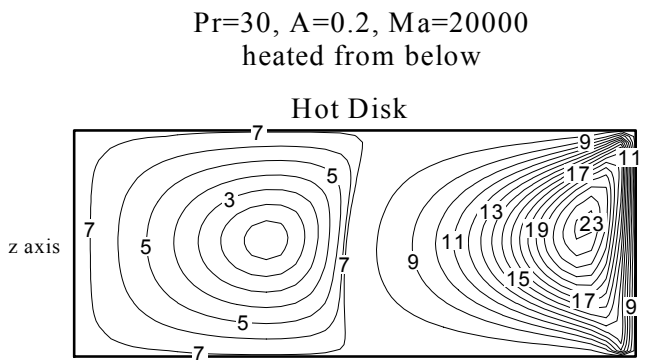

Cold Disk

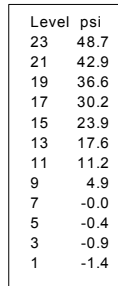

12b)

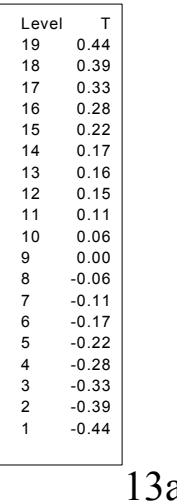

13a)

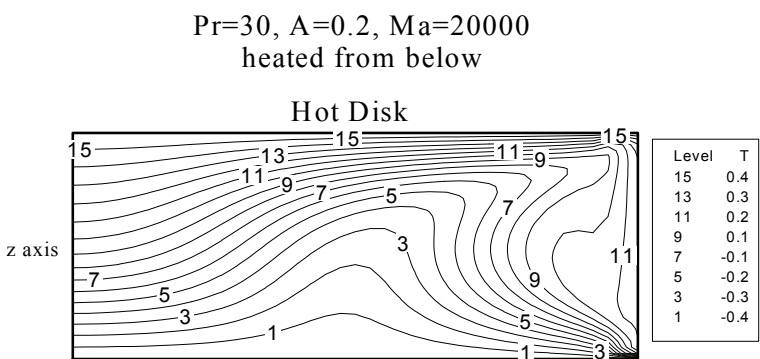

Cold Disk

$\mathrm{Pr}=30, \mathrm{~A}=0.4, \mathrm{Ma}=20000$ heated from above

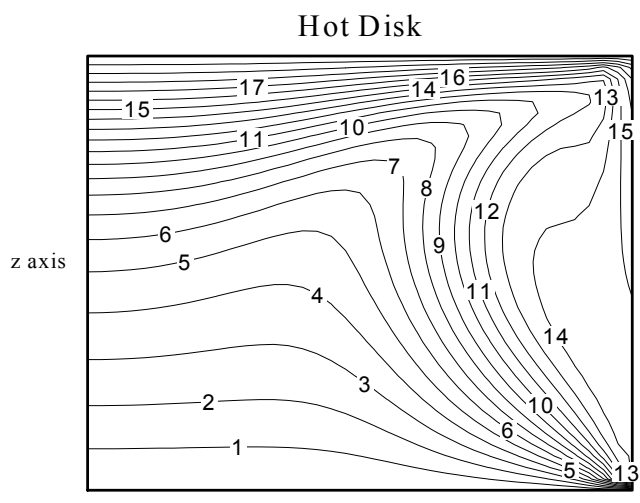

Cold Disk

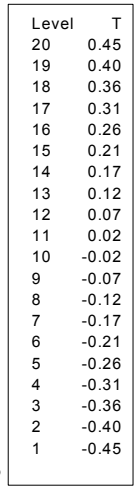

$14 a)$

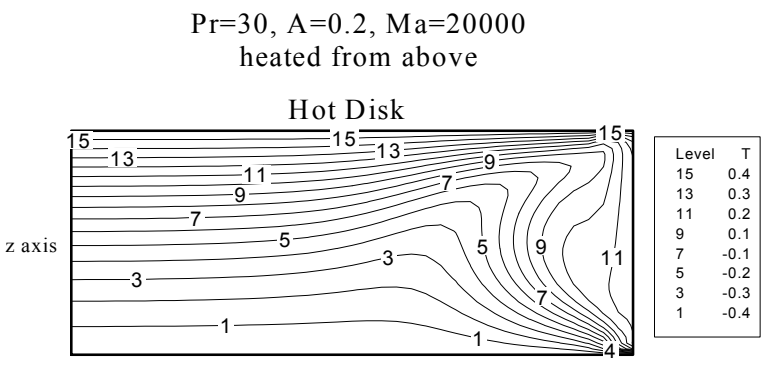

Cold Disk
$14 b)$ 


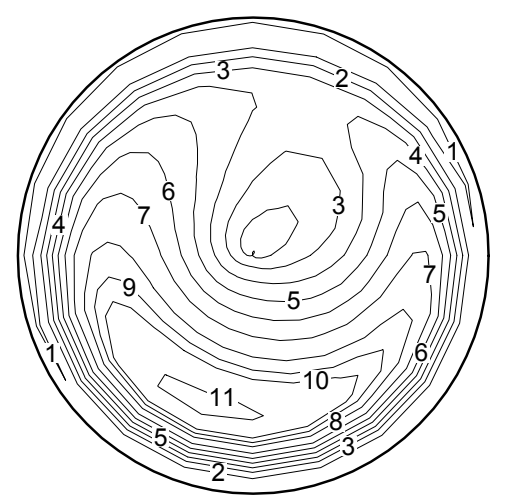

a)
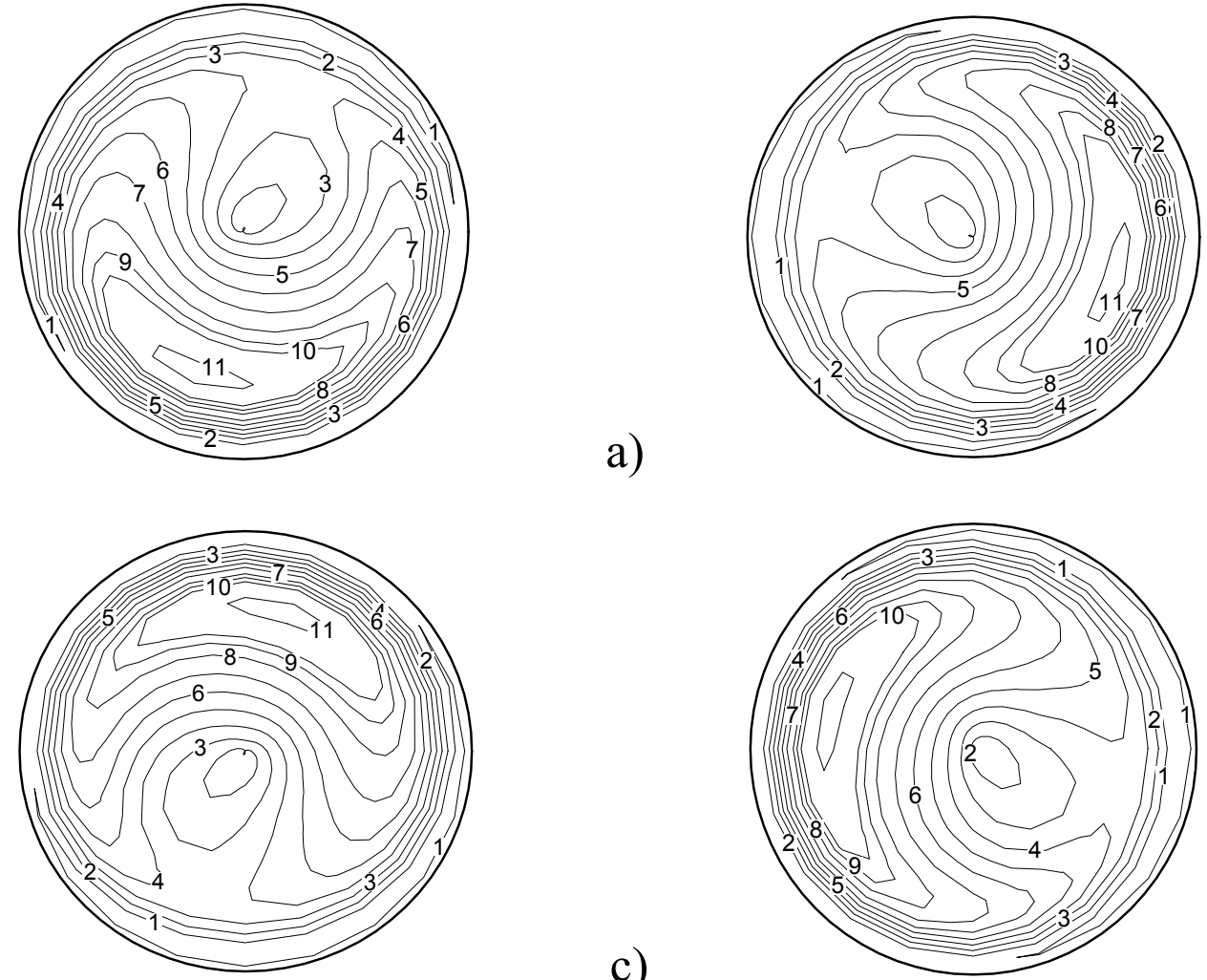

c)

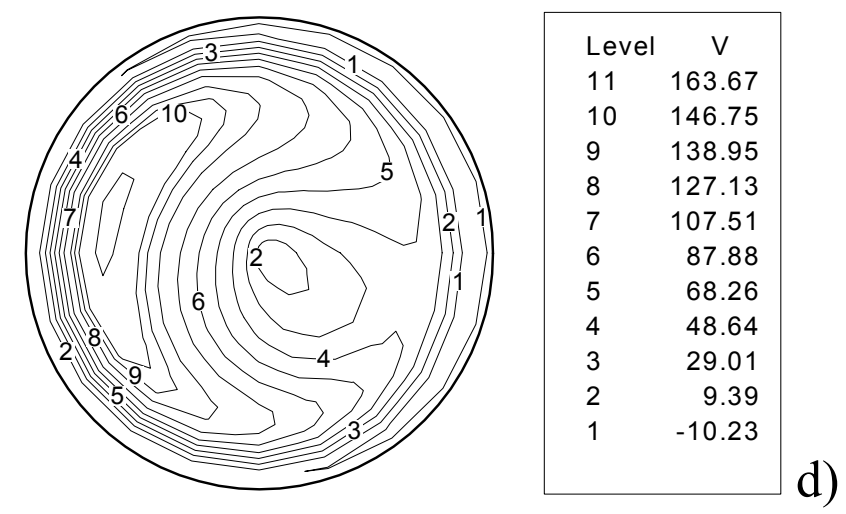

Figg. 15

Radial velocity distribution in the section $\mathrm{z}=0.75$ for $\mathrm{A}=0.4$ and traveling wave regime (bridge heated from below)

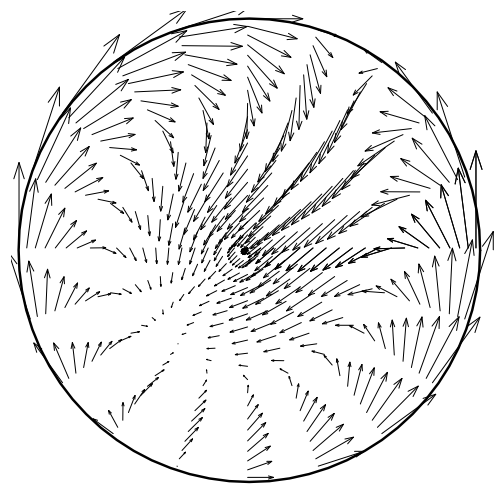

a)
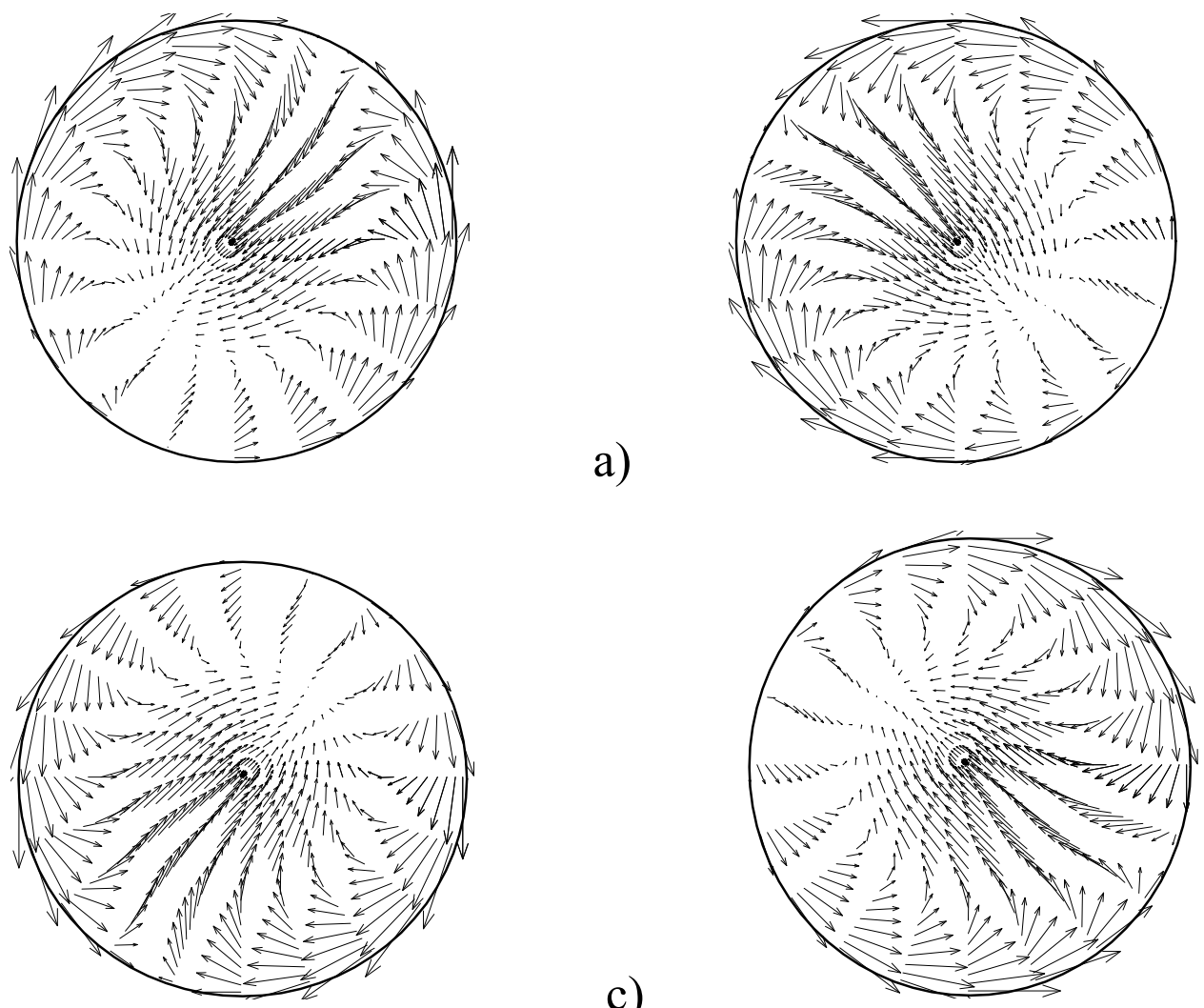

b)

c)

Figg. 16

d)

Velocity field in the section $\mathrm{z}=0.5$ for $\mathrm{A}=0.4$ and traveling wave regime (bridge heated from below) 

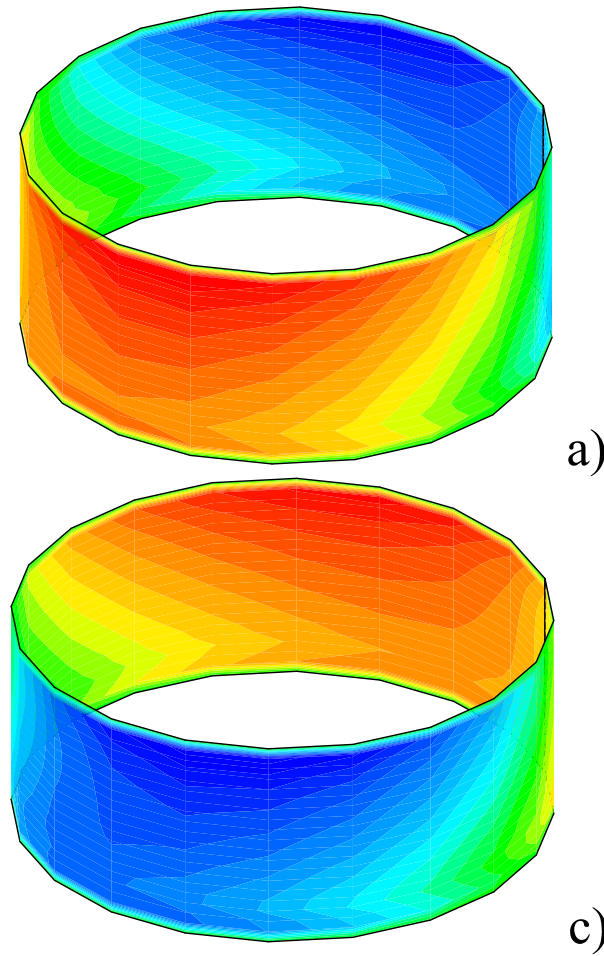

c)

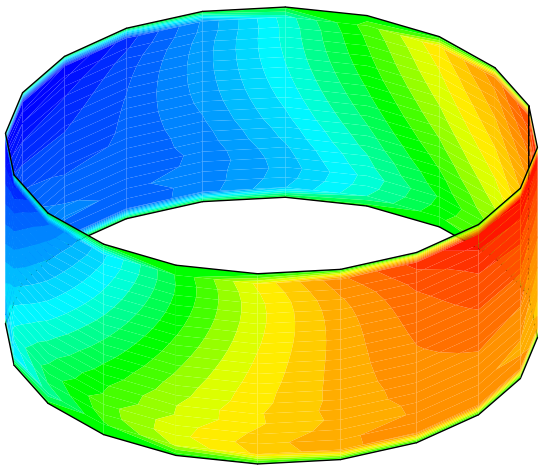

b)
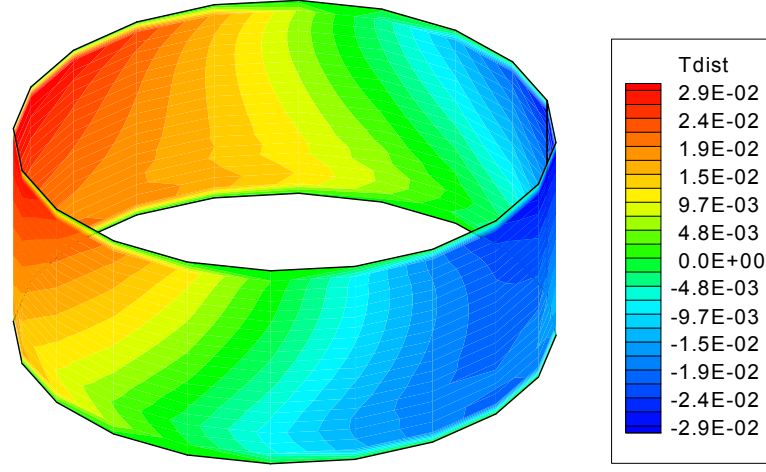

d)

Figg. 17

Temperature disturbances on the bridge surface for $\mathrm{A}=0.4$ and traveling wave regime (bridge heated from below, numerical results)

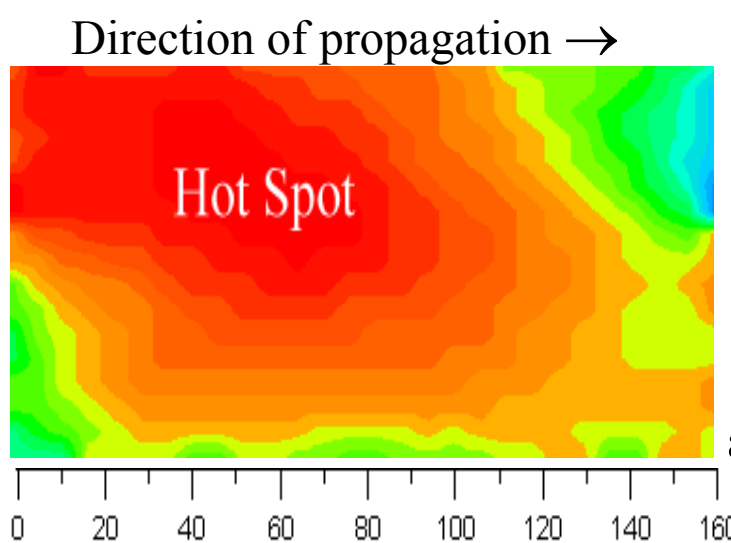

Direction of propagation $\rightarrow$

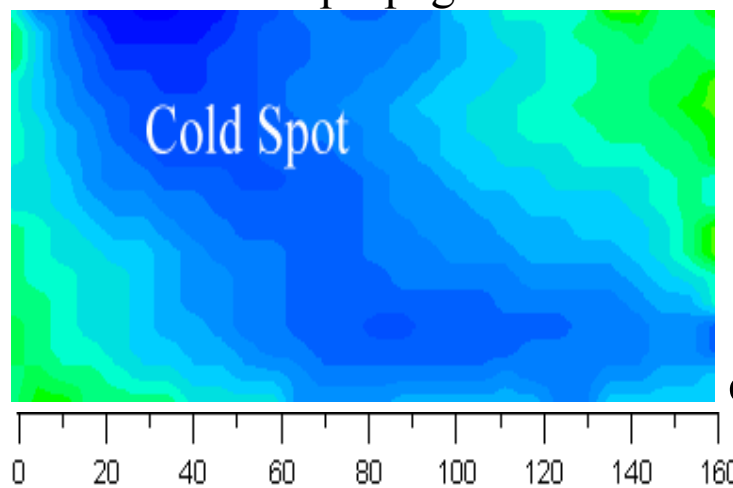

c)

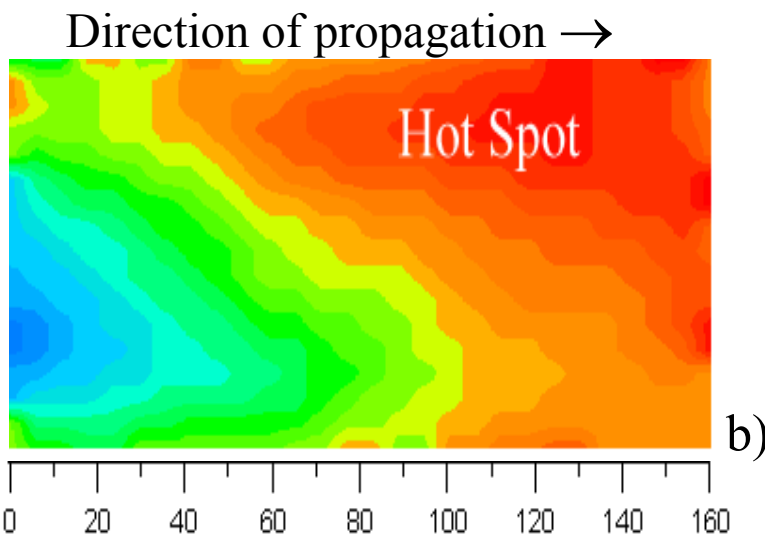

Direction of propagation $\rightarrow$

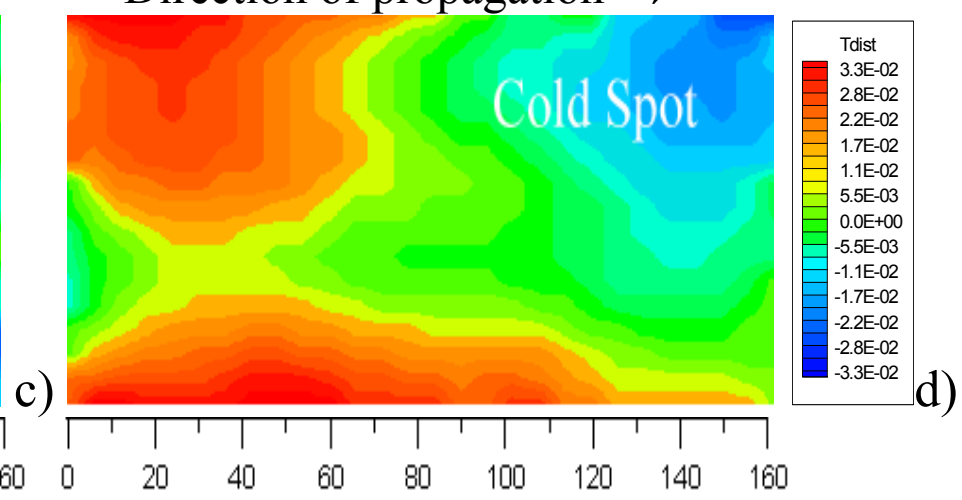

Figg. 18

Temperature disturbances on the bridge surface for $\mathrm{A}=0.4$ and traveling wave regime (bridge heated from below, experimental results) 


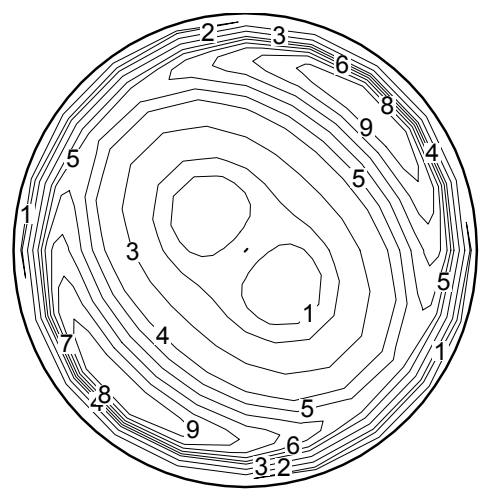

a)

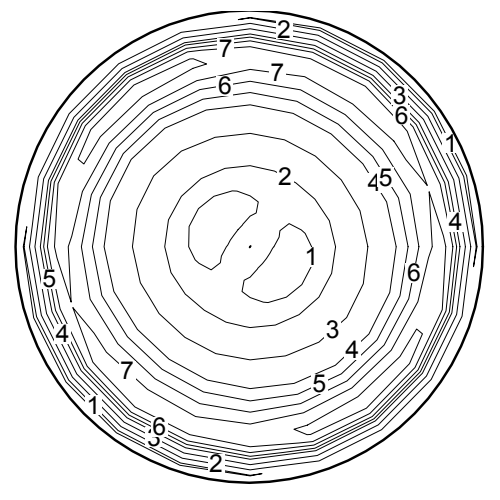

b)
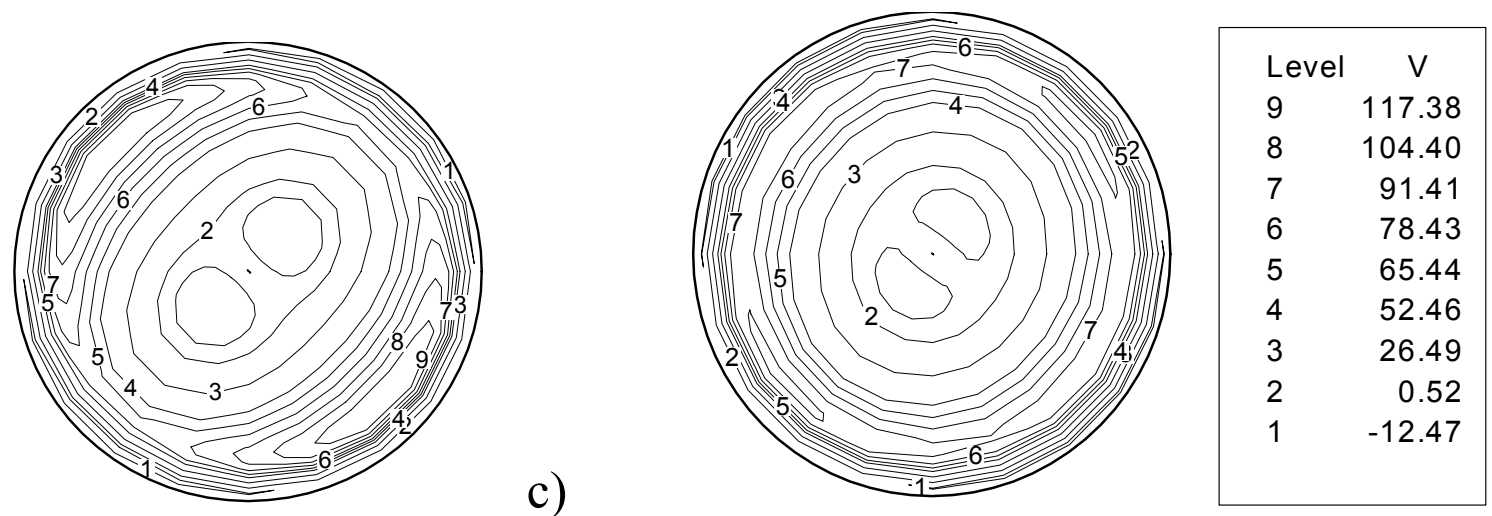

d)

Figg. 19

Radial velocity distribution in the section $\mathrm{z}=0.75$ for $\mathrm{A}=0.25$ and standing wave regime (bridge heated from below)

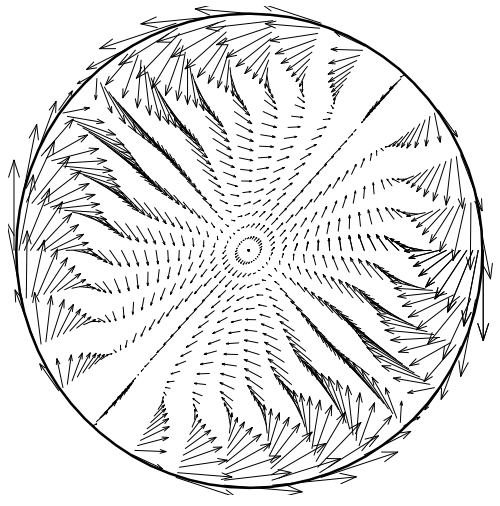

a)
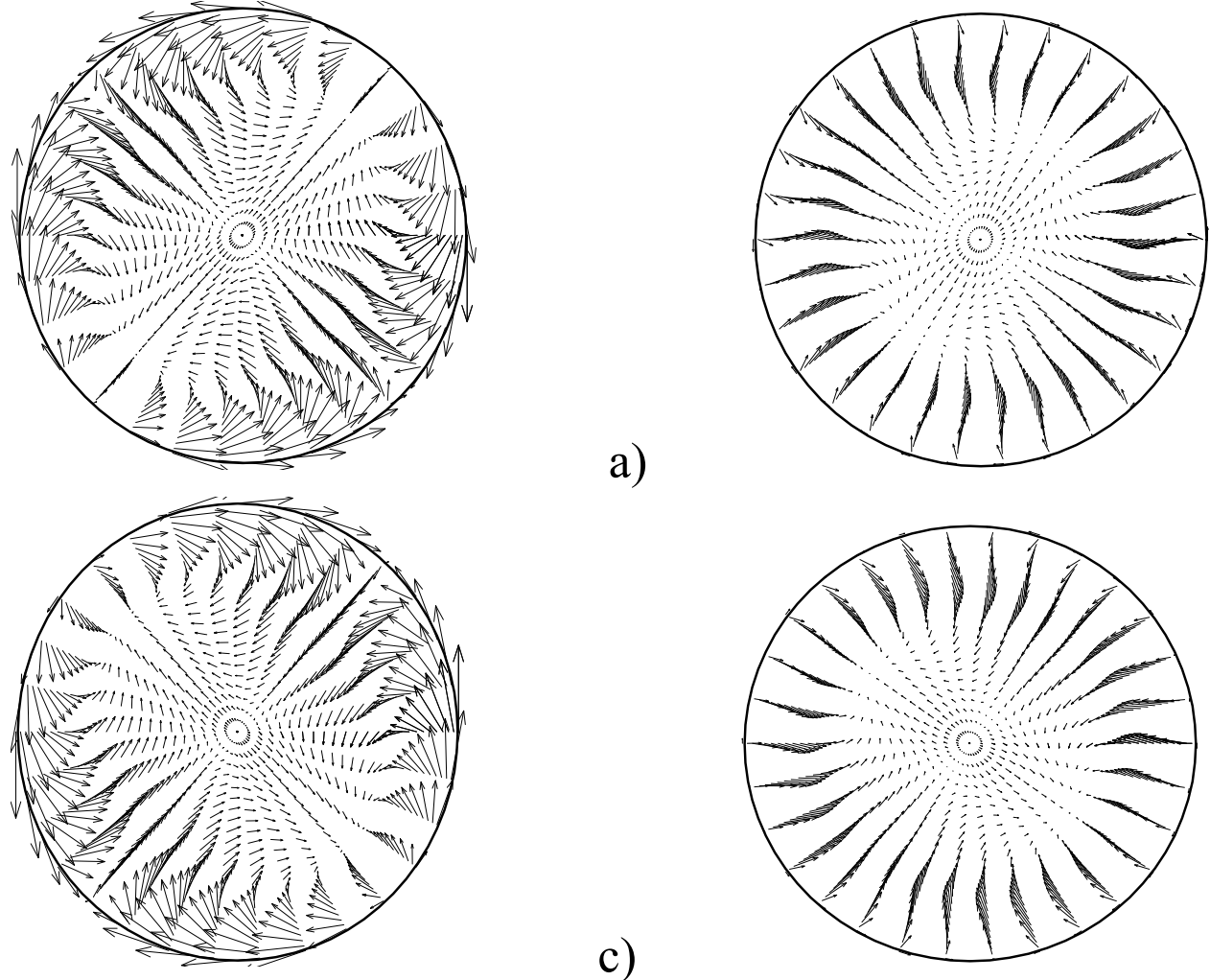

b)

c)

Figg. 20

Velocity field in the section $\mathrm{z}=0.5$ for $\mathrm{A}=0.25$ and standing wave regime (bridge heated from below) 

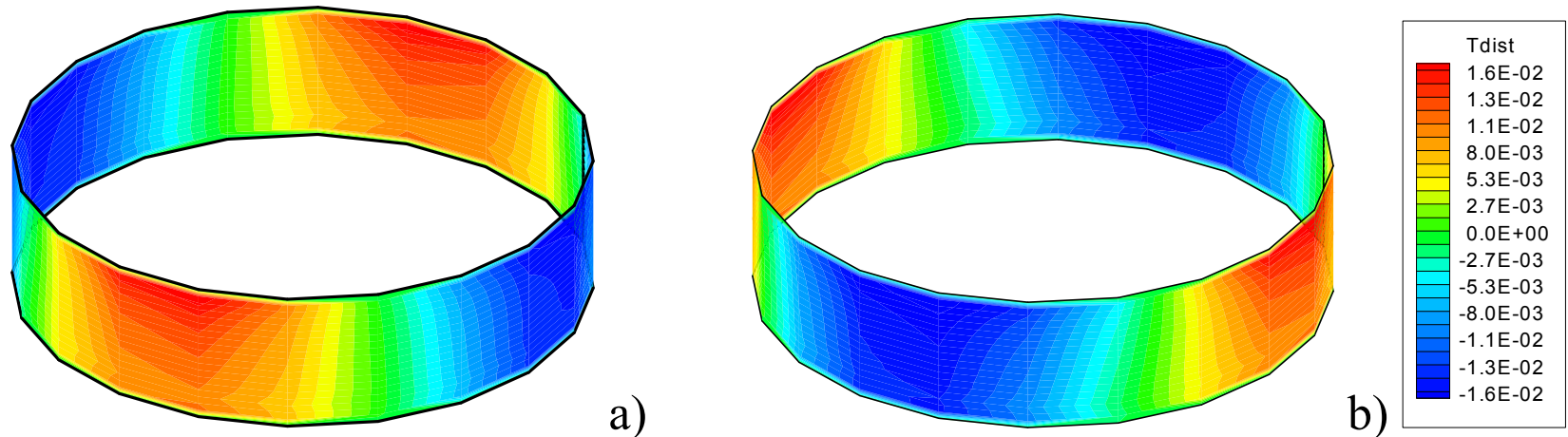

Figg. 21

Temperature disturbances on the liquid bridge surface for $\mathrm{A}=0.25$ and standing wave regime (bridge heated from below, numerical results)
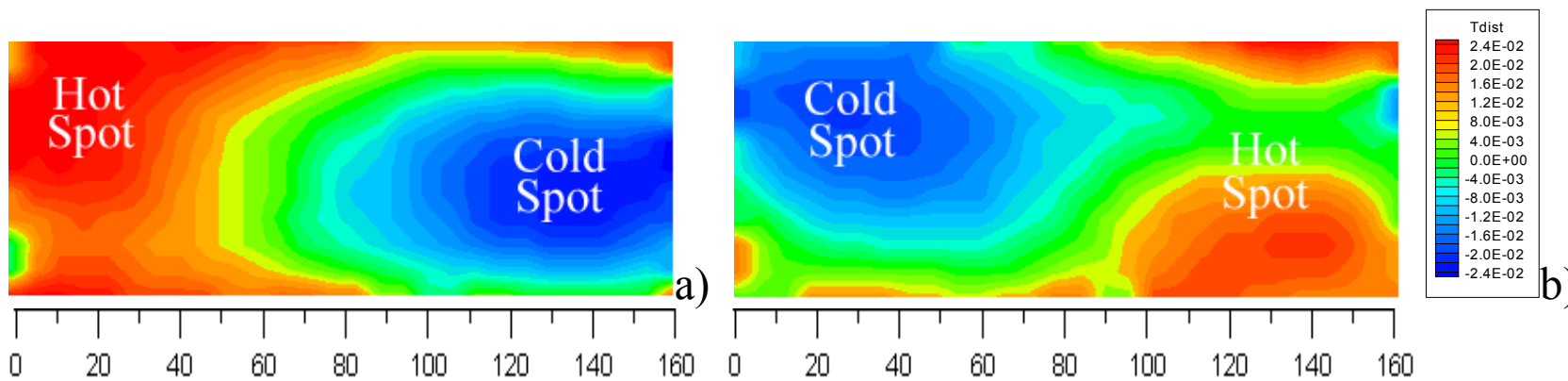

Figg. 22

Temperature disturbances on the liquid bridge surface for $\mathrm{A}=0.25$ and standing wave regime (bridge heated from below, experimental results) 


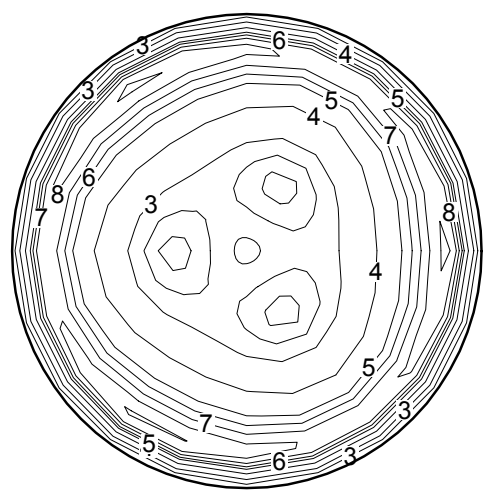

a)

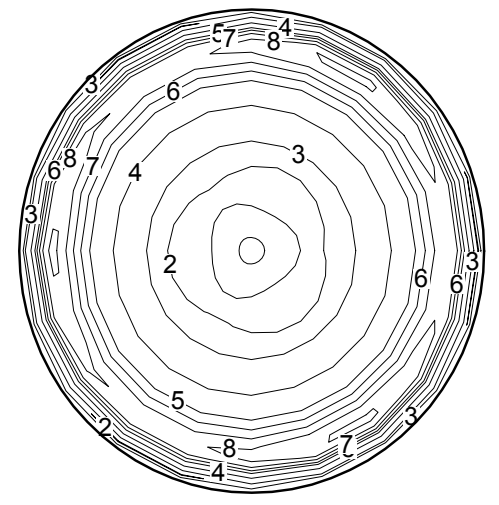

b)
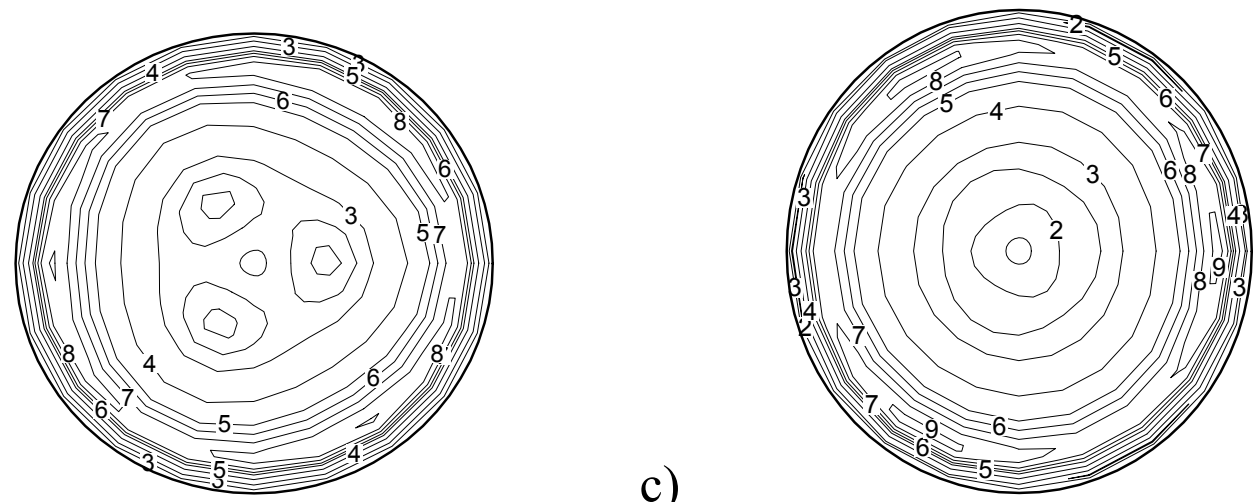

\begin{tabular}{|lr|}
\hline Level & $\mathrm{V}$ \\
9 & 104.63 \\
8 & 90.85 \\
7 & 77.06 \\
6 & 63.28 \\
5 & 49.50 \\
4 & 21.93 \\
3 & -5.64 \\
2 & -14.96 \\
1 & -19.30 \\
& \\
\end{tabular}

Figg. 23

Radial velocity distribution in the section $\mathrm{z}=0.75$ for $\mathrm{A}=0.2$ and standing wave regime (bridge heated from below)
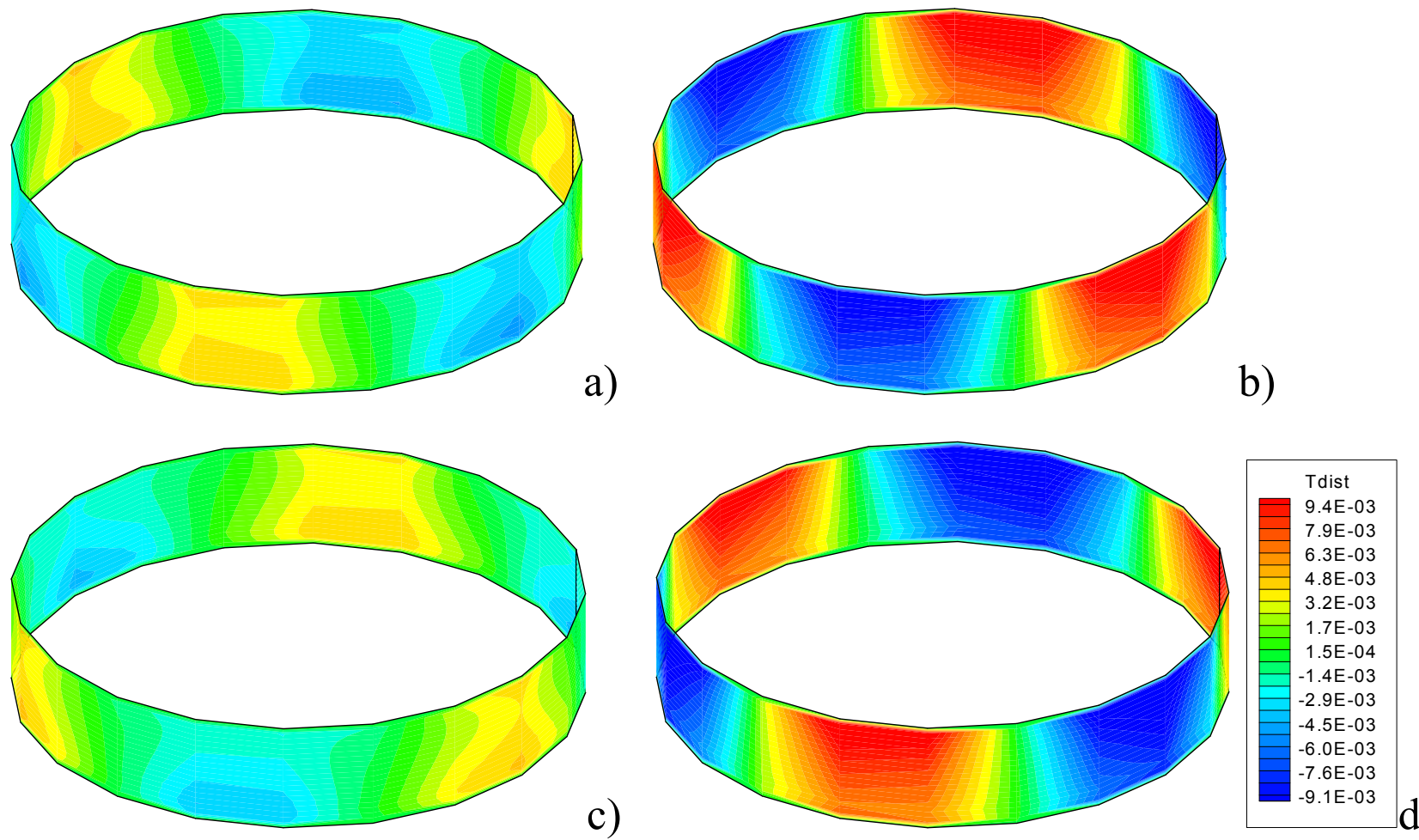

b)

c)

Figg. 24

Temperature disturbances on the liquid bridge surface for $\mathrm{A}=0.2$ and standing wave regime (bridge heated from below) 


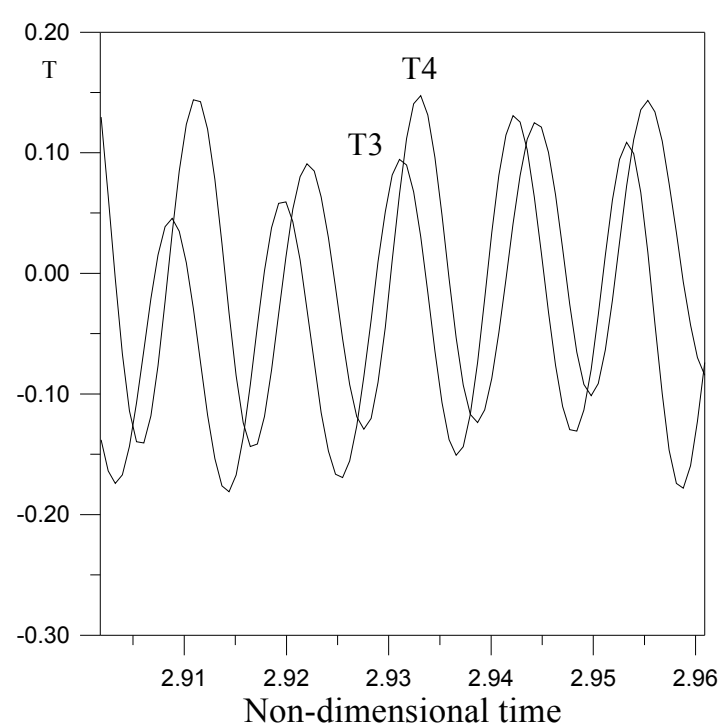

Fig. 26 a

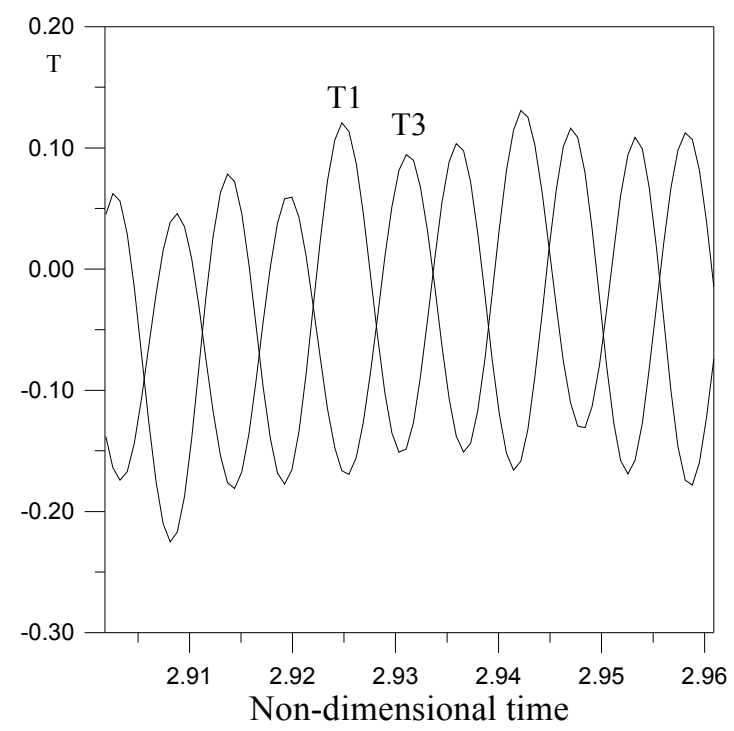

Fig. $26 \mathrm{~b}$

Figg. 26 : Experimental temperature oscillations in points having the same radial and axial coordinates located at different azimuthal positions $(\mathrm{A}=0.5$, bridge heated from below, $\mathrm{z}=0.25, \mathrm{r}=0.5$ )

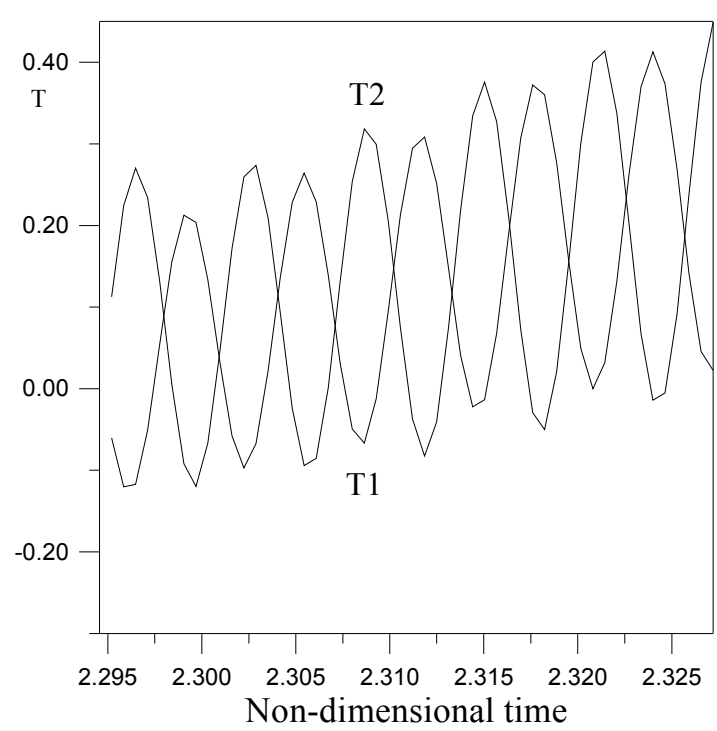

Fig. $27 \mathrm{a}$

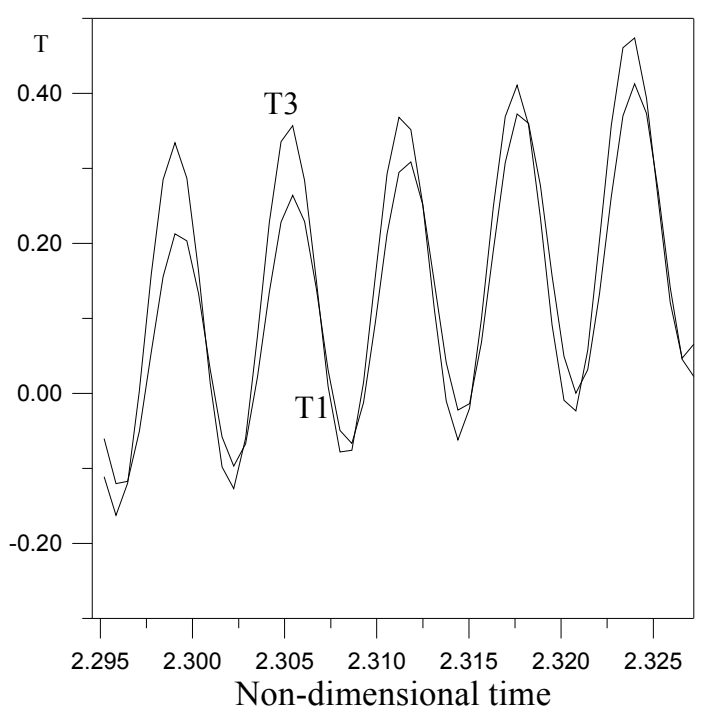

Fig. $27 \mathrm{~b}$

Figg. 27 : Experimental temperature oscillations in points having the same radial and axial coordinates located at different azimuthal positions $(\mathrm{A}=0.5$, bridge heated from above, $\mathrm{z}=0.75, \mathrm{r}=0.5$ ) 


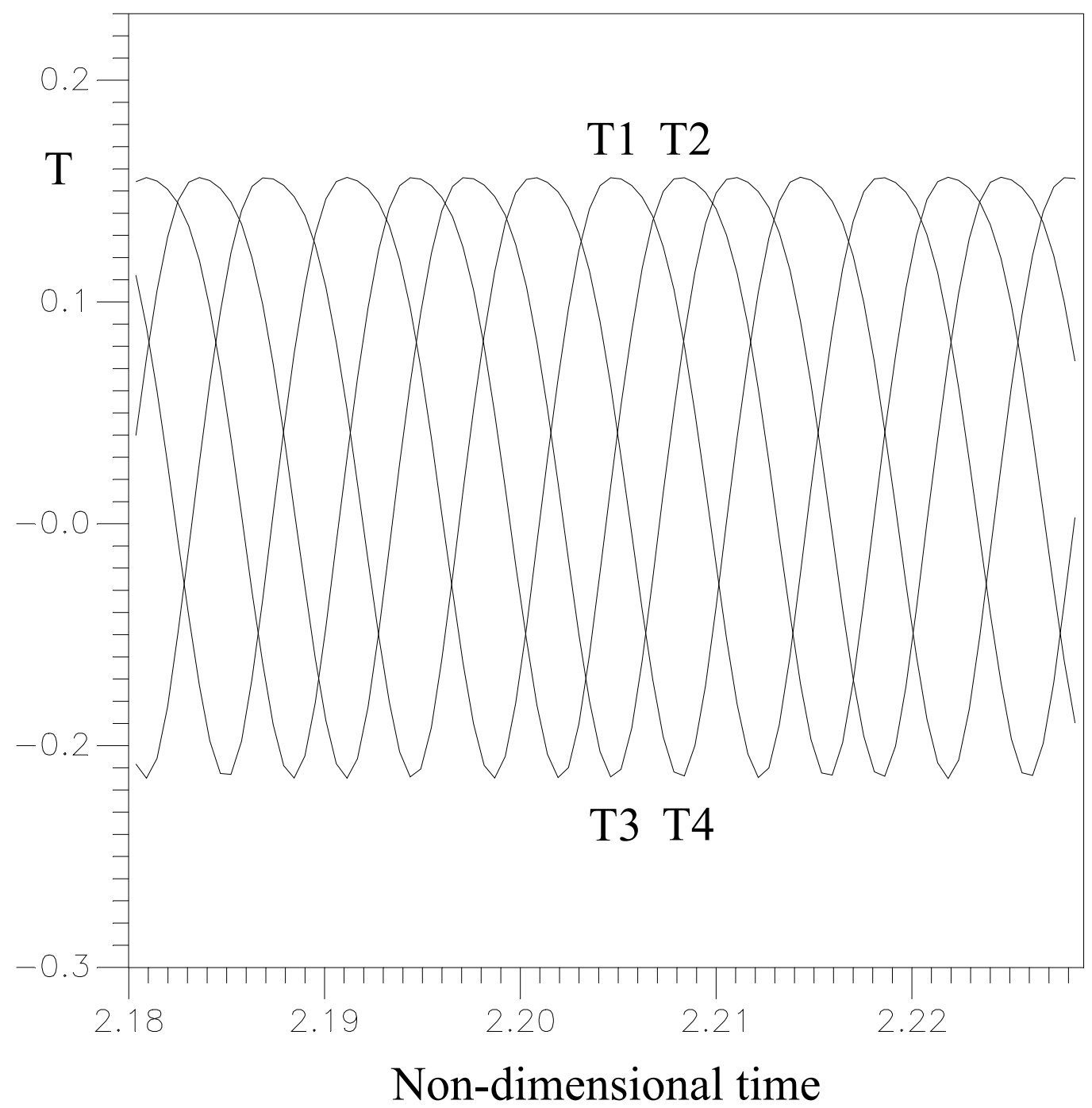

Fig. 28

Temperature oscillation in the point $\mathrm{z}=0.75, \mathrm{r}=0.5, \varphi=0$ $\mathrm{A}=0.5, \mathrm{Ma}=4.310^{4}$, bridge heated from below 